\title{
Local rigidity of certain partially hyperbolic actions of product type
}

\author{
VIOREL NIŢICĂ $\dagger$ and ANDREI TÖRÖK $\ddagger$ \\ Institute of Mathematics of the Romanian Academy, PO Box 1-764, \\ RO-70700 Bucharest, Romania
}

(Received 8 November 1999 and accepted in revised form 29 July 2000)

\begin{abstract}
We prove certain rigidity properties of higher-rank abelian product actions of the type $\alpha \times \operatorname{Id}_{N}: \mathbb{Z}^{\kappa} \rightarrow \operatorname{Diff}(M \times N)$, where $\alpha$ is (TNS) (i.e. is hyperbolic and has some special structure of its stable distributions). Together with a result about product actions of property $(T)$ groups, this implies the local rigidity of higher-rank lattice actions of the form $\alpha \times \operatorname{Id}_{\mathbb{T}}: \Gamma \rightarrow \operatorname{Diff}(M \times \mathbb{T})$, provided $\alpha$ has some rigidity properties itself, and contains a $(T N S)$ subaction.
\end{abstract}

\section{Introduction}

This paper is a contribution to the rigidity program initiated by Zimmer [Z].

The goal of the program is to classify the smooth actions of higher-rank semi-simple Lie groups and of their (irreducible) lattices on compact manifolds. It was expected that any such lattice action that preserves a smooth volume form and is ergodic can be reduced to one of the following standard models: isometric actions, linear actions on nilmanifolds, and left translations on compact homogeneous spaces. This original conjecture was disproved by Katok and Lewis (see [KL2]): by blowing up a linear nilmanifold-action at some fixed points they exhibit real-analytic, volume-preserving, ergodic lattice actions on manifolds with complicated topology.

Nevertheless, imposing additional assumptions on the action, for example, some hyperbolicity, allows for global classification results. The existence of an Anosov element in the action is used in [KLZ] to give a global classification of $\operatorname{SL}(n, \mathbb{Z})$ actions on $\mathbb{T}^{n}$ that preserve an absolutely continuous probability measure. Other global classification results for Anosov actions can be found in [GS].

Much work was also done to study perturbations of higher-rank lattices and Lie groups actions (see [H, KL1, KL2, KLZ, KS2, MQ, QY1, QY2]). In many of these papers, the $\dagger$ Current address: Department of Mathematics, University of Notre Dame, Room 370, CCMB, Notre Dame, IN 46556-5683, USA (e-mail: nitica.1@nd.edu).

\$ Current address: Department of Mathematics, University of Houston, Houston, TX 77204-3476, USA (e-mail: torok@math.uh.edu). 
existence of an Anosov element, respectively of a spanning family of directions that are hyperbolic for certain elements of the action, was essential. A first attempt to study the deformation rigidity of partially hyperbolic product actions that contain a compact factor was carried out in [NT1].

Notation. (1) Unless specified otherwise, we assume that all manifolds and maps are smooth.

By $C^{k^{-}}$we denote the class of functions that are $C^{k-\varepsilon}$ for any $\varepsilon>0$. The $C^{k-}$-topology stands for the coarsest topology for which the inclusions $C^{k-} \subset C^{k-\varepsilon}$ are continuous for each $\varepsilon>0$. However, by $C^{1^{-}}$we mean $C^{1}$.

(2) Throughout this paper by a $C^{r}$-lamination we mean a topological foliation whose leaves are $C^{r}$-submanifolds that vary continuously in the $C^{r}$-topology.

Definition. Let $\Gamma$ be a finitely generated discrete group, $M$ a compact manifold, and $\phi, \widetilde{\phi}: \Gamma \times M \rightarrow M C^{\infty}$-actions. Fix a finite set of generators $\left\{\gamma_{i}\right\}$ of $\Gamma$. We say that $\phi$ is $C^{L}$-close to $\widetilde{\phi}$ if the $C^{\infty}$-diffeomorphisms $\phi\left(\gamma_{i}\right)$ and $\widetilde{\phi}\left(\gamma_{i}\right)$ are close in the $C^{L}$-topology for all $i$. A $C^{L}$-perturbation of the action $\phi$ is a $C^{\infty}$-action $C^{L}$-close to $\phi$. A $C^{L}$-deformation of the action $\phi$ is a $C^{L}$-continuous path of $C^{\infty}$-actions $\phi_{t}, 0 \leq t \leq 1$, with $\phi_{0}=\phi$. An action $\phi$ is said to be $C^{L, K}$-locally rigid if any $C^{L}$-perturbation of $\phi$ contained in a sufficiently small $C^{L}$-neighborhood of $\phi$ is conjugated to $\phi$ by a $C^{K}$-diffeomorphism which is $C^{0}$-close to the identity. An action $\phi$ is said to be $C^{L, K}$-deformation rigid if any $C^{L}$-deformation of $\phi$ contained in a sufficiently small $C^{L}$-neighborhood of $\phi$ is conjugated to $\phi$ by a continuous path of $C^{K}$-diffeomorphisms $C^{0}$-close to the identity.

We are interested in rigidity results for partially hyperbolic actions that have a trivial factor. Deformation rigidity results were obtained in [NT1]. The main goal of this paper is to prove the local rigidity of certain actions of this type.

Let $\pi$ be the standard linear action of $\operatorname{SL}(n, \mathbb{Z})$ on the $n$-dimensional torus $\mathbb{T}^{n}$. The holonomy considerations used in this paper lead to the following improvement upon the main result of [NT1] (see the end of $\S 4$ for the proof).

THEOREM 1.1. Let $n \geq 3$ and $d \geq 1$ be integers. Let $\rho$ be the action of $\operatorname{SL}(n, \mathbb{Z})$ on $\mathbb{T}^{n+d}=\mathbb{T}^{n} \times \mathbb{T}^{d}$ given by $\rho(A)(x, y)=(\pi(A) x, y), x \in \mathbb{T}^{n}, y \in \mathbb{T}^{d}, A \in \operatorname{SL}(n, \mathbb{Z})$. Then, for any integer $K \geq 1$, the action $\rho$ is $C^{5, K^{-}}$-deformation rigid.

One special case of our new result is a strengthening of Theorem 1.1 when the fibers are one-dimensional (see the proof after that of Theorem 3.3).

THEOREM 1.2. Let $\Gamma \subset S L(n, \mathbb{Z})$ be a subgroup of finite index and let $\rho$ be the action of $\Gamma$ on $\mathbb{T}^{n+1}=\mathbb{T}^{n} \times \mathbb{T}$ given by $\rho(A)(x, y)=(\pi(A) x, y), x \in \mathbb{T}^{n}, y \in \mathbb{T}, A \in \Gamma$. Then, for $n \geq 3$ and any integer $K \geq 1$, the action $\rho$ is $C^{2, K^{-}}$-locally rigid.

More examples are given in Corollary 3.4 and the remarks following it.

The main part of Theorem 1.1, proved in [NT1], is based on three results in hyperbolic dynamics: a generalization of Livsic's cohomological results to cocycles with values in diffeomorphism groups, a non-commutative version of the Anosov closing lemma, and a version of the Hirsch-Pugh-Shub structural stability theorem. 
Note that Theorem 1.2 does not imply that the action $\rho$ is $C^{2, \infty}$-locally rigid because the size of the $C^{2}$-neighborhood guaranteed by the theorem depends on $K$. The main ingredients of the proof are two rigidity results: one concerns (TNS) abelian actions, the other actions of property-( $T)$ groups. This approach obtains the rigidity of partiallyhyperbolic actions of product type from the (known) rigidity of hyperbolic actions.

It has been noticed for some time that many Anosov actions of abelian groups display an array of rigidity properties. The vanishing of the first cohomology groups with coefficients in $\mathbb{R}^{n}$ was noticed for the first time in [KS1]. This result is extended to cohomology groups with coefficients in a matrix Lie group in [KNT]. Local and global rigidity results for abelian Anosov actions are proved in [KL1] and [KS2].

As a consequence of the Hirsch-Pugh-Shub structural stability theorem (see [HPS] and Theorem 2.1), small perturbations of abelian partially hyperbolic actions of product type are conjugated to skew products of abelian Anosov actions by cocycles with coefficients in diffeomorphism groups. Given the previous results, it is natural to expect certain rigidity phenomena to appear for these actions as well.

A basic fact proved in this paper is that for certain higher-rank abelian partially hyperbolic actions of product type, the sum of the stable and unstable distributions of any regular element of the perturbation is integrable. In view of the situation for Lie group valued cocycles (see $[\mathbf{K N T}]$ ), one might expect the leaves of the integral lamination to be closed manifolds covering the base simply, thus obtaining a conjugacy between the perturbation and a product action. This amounts to showing that the only small solutions of a particular system of equations in Diff (or Homeo), determined by the holonomy of the integral lamination, are the trivial ones. We obtain the above-mentioned conclusion under the supplementary assumption that some regular element of the perturbation has a pointwise fixed center leaf. It is not clear to us how to remove this additional assumption.

Namely, one has to deal with the following question.

Question. Let $T: M \rightarrow M$ be an Anosov diffeomorphism on the compact manifold $M$. Let $N$ be a compact manifold and $f$ a partially hyperbolic diffeomorphism of $M \times N$ which is $C^{1}$-close to $T \times \operatorname{Id}_{N}$. Assume that the stable and unstable foliations of $f$ are jointly integrable. Does it follow that the diffeomorphism $f$ is conjugated to $T \times S$, where $S \in \operatorname{Homeo}(N)$ ? That is, does the foliation integrating the stable and unstable foliations of $f$ have closed leaves?

Theorem 1.2 is obtained as follows. Consider first the restriction of the action $\rho$ to a diagonalizable abelian subgroup of rank $n-1$ of $\Gamma$. The existence of a pointwise fixed center fiber is ensured by a theorem of Stowe [St1, St2], first used in this context by Hurder $[\mathbf{H}]$. Applying the rigidity result to this abelian action, we obtain a conjugacy between the original abelian action and its perturbation. Using the property $(T)$, we show that this conjugacy reduces the $\Gamma$-action to a family of perturbations of hyperbolic actions.

This paper has the following structure: in $\$ 2$ we recall a few facts from the theory of partially hyperbolic diffeomorphisms and the definition of a (TNS) action, respectively of property $(T)$. In $\S 3$ we present the main results, and in $\S \S 4$ and 5 we give the proofs. 
2. Preliminaries

We recall first the definition of a partially hyperbolic diffeomorphism.

If $T$ is a linear transformation between two normed linear spaces, the norm and the conorm of $T$ are defined by

$$
\|T\|=\sup \{\|T v\| ;\|v\|=1\} \quad \text { and } \quad m(T)=\inf \{\|T v\| ;\|v\|=1\} .
$$

Let $X$ be a compact, connected, boundaryless manifold. A $C^{1}$-diffeomorphism $f$ : $X \rightarrow X$ is called partially hyperbolic if the derivative $T f: T X \rightarrow T X$ leaves invariant a continuous splitting $T X=E^{s} \oplus E^{c} \oplus E^{u}, E^{s} \neq 0 \neq E^{u}$, such that, with respect to a fixed Riemannian metric on $T X, T f$ expands $E^{u}, T f$ contracts $E^{s}$, and the inequalities

$$
\left\|T_{p}^{s}\right\|<m\left(T_{p}^{c} f\right), \quad\left\|T_{p}^{c} f\right\|<m\left(T_{p}^{u} f\right)
$$

are true for all $p \in X$. If the center bundle $E^{c}=0$, then $f$ is called an Anosov (or hyperbolic) diffeomorphism.

Assume that the partially hyperbolic $C^{r}$-diffeomorphism $f$ leaves invariant a $C^{1}$ lamination $\mathcal{L}$ tangent to the central direction $E^{c}$. We say that $f$ is $r$-normally hyperbolic at $\mathcal{L}$ if for all $p \in X$ and $0 \leq k \leq r$ one has

$$
m\left(T_{p}^{u} f\right)>\left\|T_{p}^{c} f\right\|^{k} \quad \text { and } \quad\left\|T_{p}^{s} f\right\|<m\left(T_{p}^{c} f\right)^{k} .
$$

The partially hyperbolic diffeomorphism $f$ is said to satisfy the rth-order centerbunching conditions if for all $p \in X$ and $0 \leq \ell \leq r$

$$
\left\|T_{p}^{s} f\right\|\left\|T_{p}^{c} f\right\|^{\ell}<m\left(T_{p}^{c} f\right) \quad \text { and } \quad\left\|T_{p}^{c} f\right\|<m\left(T_{p}^{u} f\right) m\left(T_{p}^{c} f\right)^{\ell} .
$$

We recall the results of [HPS, Theorems 6.1, 6.8, 7.1, 7.2] and [PSW, Theorem B] about partially hyperbolic $\mathbb{Z}$-actions and their small perturbations. We describe only the case that will be of interest in the following, and skip the description of the terms that are self-explanatory. In the case of hyperbolic diffeomorphisms, we have the classical results of Anosov [A]. See also the Remark following Theorem 2.1.

THEOREM 2.1. ([HPS]) Let $X$ be a compact manifold and $f \in \operatorname{Diff}^{r}(X), r \geq 1, a$ diffeomorphism which is $r$-normally hyperbolic at a $C^{r}$-lamination $\mathcal{L}_{f}$ having compact leaves.

(1) Through each leaf of $\mathcal{L}_{f}$ there exists a $C^{r}$ center-stable manifold. The center-stable manifold through $x \in X$ consists of those points whose forward $f$-orbit does not stray away from the orbit of $\mathcal{L}_{f}(x)$. Hence, since the leaves of the lamination $\mathcal{L}_{f}$ are compact, each center-stable manifold is a union of center leaves; the center-stable manifolds form the center-stable lamination $W^{c s}(f)$. A similar statement holds for the center-unstable lamination, $W^{c u}(f)$.

(2) There exists a $C^{r}$ stable lamination $W^{s}(f)$ whose leaves lie in those of $W^{c s}(f)$. The points of a stable leaf are characterized by sharp forward asymptoticity. If $f$ satisfies the $(r-1)$ th order center-bunching conditions, then the stable distribution is $C^{r-1}$ on each center-stable leaf. In particular, the holonomy maps determined by the stable lamination inside the center-stable leaves are $C^{r-1}$. A similar statement holds for the unstable lamination, $W^{u}(f)$. 
(3) If $g \in \operatorname{Diff}^{r}(X)$ is $C^{1}$-close to $f$, then $g$ is $r$-normally hyperbolic at a unique $C^{r}$ lamination $\mathcal{L}_{g}$, and the stable, unstable, and center laminations of $g$ converge in $C^{r}$ to those of $f$ as $g$ converges to $f$ in the $C^{r}$-topology. The stable (unstable) holonomy maps within the center-stable (respectively, center-unstable) leaves of $g$ converge in $C^{r-1}$ to those of $f$, as g converges in $C^{r}$ to $f$.

(4) Moreover, if $\mathcal{L}_{f}$ is a $C^{r}$-foliation, then in the case (3) there exists a leaf-conjugacy $H \in \operatorname{Homeo}(X)$ between $\left(f, \mathcal{L}_{f}\right)$ and $\left(g, \mathcal{L}_{g}\right): H$ maps the leaves of $\mathcal{L}_{f}$ to those of $\mathcal{L}_{g}$ and $\mathcal{L}_{g}(H \circ f(x))=\mathcal{L}_{g}(g \circ H(x)) . H$ is a $C^{r}$-diffeomorphism of each leaf of $\mathcal{L}_{f}$ onto its image, varying continuously in $C^{r}$ with the leaf. For $x \in X, \mathcal{L}_{g}(H(x))$ is uniquely characterized by the fact that its g-orbit does not stray away from the $f$-orbit of $\mathcal{L}_{f}(x)$. Modulo the choice of a normal bundle to $\mathcal{L}_{f}, H$ is uniquely determined. If $g$ converges to $f$ in the $C^{r}$-topology then $H$ converges to the identity in the $C^{r}$-topology along the leaves of $\mathcal{L}_{f}$ and to $\operatorname{Id}_{X}$ in $C^{0}$.

Here 'never strays away' means that $g^{n}\left(\mathcal{L}_{g}(H(x))\right)$ stays within a tubular neighborhood of predetermined small size of $f^{n}\left(\mathcal{L}_{f}(x)\right)$, for each $n \in \mathbb{Z}$.

Remark. The statement in (2) about the smoothness of the stable distribution along the leaves of $W^{c s}$ follows from the $C^{r}$-section theorem [HPS, Theorem 3.5] (applied in this case for $(r-1))$. The compactness of the base space can be replaced by the appropriate uniformities. The continuous dependence of these holonomies described in (3) follows from a straightforward generalization of the similar continuity contained in the $C^{r}$-section theorem. Theorem B of [PSW] proves that the holonomy of $W^{s}$ inside $W^{c s}$ is $C^{r-1}$ under milder conditions.

The crucial property on which the rigidity results for abelian actions are based is that of a (TNS) action, introduced by Katok (see [KNT]).

Definition. Let $\alpha: \mathcal{A} \times X \rightarrow X$ be an action of $\mathcal{A}=\mathbb{Z}^{\kappa}$ on a compact manifold $X$. We say that the action $\alpha$ is totally non-symplectic, or (TNS), if there is a family $S$ of partially hyperbolic elements in $\mathcal{A}$ and a continuous splitting of the tangent bundle $T X=\oplus_{i=1}^{\ell} E_{i}$ into $\mathcal{A}$-invariant distributions such that:

(i) the stable and unstable distributions of any element in $S$ are direct sums of a subfamily of the $E_{i}$ 's;

(ii) any two distributions $E_{i}$ and $E_{j}, 1 \leq i, j \leq \ell$, are included in the stable distribution of some element in $S$.

Remarks. (1) It is easy to see that given a (TNS) action, one can assume that $S$ consists only of Anosov elements.

(2) Given a $(T N S)$ action described by $S \subset \mathcal{A}$ with all elements of $S$ Anosov and a splitting $T X=\oplus_{i=1}^{\ell} E_{i}$, one can replace the distributions $\left\{E_{i}\right\}$ by the non-zero intersections $\bigcap_{a \in S} E^{\sigma(a)}(a)$, where $\sigma(a) \in\{u, s\}$. Indeed, denote the new splitting by $T X=\oplus_{i=1}^{k} F_{i}$. It obviously satisfies (i), and (ii) can be checked as follows: given $F_{i}$ and $F_{j}$, there are $1 \leq i^{\prime}, j^{\prime} \leq \ell$ such that $E_{i^{\prime}} \subset F_{i}$ and $E_{j^{\prime}} \subset F_{j}$ and $a \in S$ such that $E_{i^{\prime}}, E_{j^{\prime}} \subset E^{s}(a)$; then $F_{i}, F_{j} \subset E^{s}(a)$, by the choice of the new splitting.

(3) If the action $\alpha$ is linear, and $L_{j}: \mathbb{Z}^{\kappa} \rightarrow \mathbb{R}$ are its Lyapunov exponents (i.e. the logarithms of the absolute values of the eigenvalues of the matrix corresponding to the 
derivative of the action; see [KS3]), then the (TNS) property can be characterized by:

$$
\text { if } L_{j}=c L_{i} \text { for some constant } c \text {, then } c>0 \text {. }
$$

In the following we will use the splitting given by Remark (2) above. Note that it is given by integrable distributions. We call the corresponding laminations minimal.

Several examples of $(T N S)$ actions are presented in [KNT, §7]. We also discuss an example in $\S 3$ of this paper, after Corollary 3.4.

Call an element $a \in \mathcal{A}$ regular if $\alpha(a)$ is hyperbolic.

Recall the definition of Kazhdan's property ( $T$ ) (see Proposition 1.14 in [HV]).

Definition. A discrete group $\Gamma$ has the property $(T)$ if there exists a finite set $S \subset \Gamma$ and $\delta>0$ such that any unitary representation $\pi: \Gamma \rightarrow \mathcal{U}(H)$ that has a non-zero $(\delta, S)$ invariant vector has a non-zero invariant vector as well. (A vector $\xi \in H$ is $(\delta, S)$-invariant if $\|\pi(a) \xi-\xi\| \leq \delta\|\xi\|$ for all $a \in S$.)

Finally, we introduce one more piece of notation, motivated by the examples we are going to consider.

Notation. Given a partially hyperbolic diffeomorphism, by a horizontal lamination we mean a $C^{1}$-lamination whose leaves are transverse to the center distribution. In particular, by the horizontal foliation of a product action $\alpha \times \operatorname{Id}_{N}$ on $M \times N$ we mean the foliation with leaves $M \times\{y\}, y \in N$.

\section{Results}

To state our results in full generality we need the following definition.

Definition. Let $\Gamma$ be a discrete group, $M$ a compact manifold, $K, L \geq 1$, and $\alpha: \Gamma \rightarrow$ $\operatorname{Diff}^{K}(M)$ an action. The action $\alpha$ is called continuously $C^{L, K}$-locally rigid if it is $C^{L, K}$-locally rigid, and the conjugacy varies continuously in the $C^{k^{-}}$topology when the perturbation varies continuously within a compact set in the $C^{k}$-topology, for $1 \leq k \leq K$.

Examples of continuously locally rigid actions are described before Corollary 3.4.

It is not hard to see that 'bounded' $C^{L, K}$-local rigidity implies continuous $C^{L, K}$-local rigidity. We do not know, however, whether either of them are implied by $C^{L, K}$-local rigidity.

The two main results of this paper are the following.

THEOREM 3.1. Let $\mathcal{A}=\mathbb{Z}^{\kappa}(\kappa \geq 2), M=\mathbb{T}^{m}, N$ be a compact manifold and $\alpha: \mathcal{A} \rightarrow \operatorname{Diff}^{K+1}(M)$ be a $(T N S)$ action, where $K \geq 0$ is fixed. Consider $\rho: \mathcal{A} \rightarrow$ $\operatorname{Diff}^{K+1}(M \times N)$ given by $\rho(a):=\alpha(a) \times \operatorname{Id}_{N}$.

(a) Any $C^{K+1}$-action $\tilde{\rho}$ that is $C^{1}$-close to $\rho$ has an invariant horizontal lamination $\tilde{\mathcal{H}}$ which has $C^{(K+1)^{-}}$leaves and $C^{K}$-holonomy between the center leaves of $\widetilde{\rho}$. The closeness depends on $(K+1)$-normal hyperbolicity and $K$ th-order center-bunching. As $\tilde{\rho}$ converges to $\rho$ in $C^{K+1}, \tilde{\mathcal{H}}$ converges to $\mathcal{H}$ in $C^{(K+1)^{-}}$and the holonomy converges uniformly in $C^{K}$ to the identity on each center leaf.

(b) Assume, moreover, that for some regular element $a \in \mathcal{A}$ there is a center leaf which is pointwise fixed by $\widetilde{\rho}(a)$. Then, for $\widetilde{\rho} C^{1}$-close enough to $\rho$, there is a homomorphism 
$\pi: \mathcal{A} \rightarrow \operatorname{Diff}^{K}(N)$ such that $\tilde{\rho}$ is conjugated to the product action $\alpha \times \pi$ by a map $h \in \operatorname{Homeo}(M \times N)$ :

$$
\widetilde{\rho}\left(a^{\prime}\right)=h\left(\alpha\left(a^{\prime}\right), \pi\left(a^{\prime}\right)\right) h^{-1}, \quad a^{\prime} \in \mathcal{A} .
$$

$h(x, \cdot)$ is a $C^{K}$-diffeomorphism onto its image for each $x \in M$ and varies continuously in $C^{K}$ with $x$. The lamination $\widetilde{\mathcal{H}}$ is the image through $h$ of the invariant horizontal foliation $\mathcal{H}$ of $\rho$. If $\tilde{\rho}$ converges to $\rho$ in $C^{1}$, then $h$ converges to $\operatorname{Id}_{M \times N}$ in $C^{0}$.

(c) Let $K \geq 1$ and assume that the action $\alpha$ is continuously $C^{L^{-}, K}$-locally rigid for some $K+1 \geq L \geq 1$. If the conditions of $(b)$ hold, $\tilde{\rho}$ is $C^{L}$-close to $\rho$, and the corresponding homomorphism $\pi: \mathcal{A} \rightarrow \operatorname{Diff}^{K}(N)$ is trivial (i.e. $h$ conjugates $\tilde{\rho}$ and $\rho)$, then $h(\cdot, y): M \rightarrow M \times N$ is $C^{K}$ and varies continuously in $C^{K-}$ with $y$. Hence, by Journé's theorem (see Theorem 4.5), $h \in \operatorname{Diff}^{K^{-}}(M \times N)$. If $\tilde{\rho}$ converges to $\rho$ in $C^{L+1}$ then $h$ converges to $\operatorname{Id}_{M \times N}$ in $C^{L^{-}}$.

Remark. Given a $C^{K+1}$ cocycle $\beta: \mathcal{A} \times M \rightarrow \operatorname{Diff}^{K+1}(N)$ over the action $\alpha$, one can construct a skew-product action $\widetilde{\rho}: \mathcal{A} \rightarrow \operatorname{Diff}^{K+1}(M \times N)$ by

$$
\tilde{\rho}(a)(x, y)=(\alpha(a), \beta(a, x)(y)), \quad \text { where } x \in M, y \in N .
$$

The conclusion of Theorem 3.1(b) is equivalent to the fact that the cocycle $\beta$ is cohomologous to a constant cocycle. Hence, under the additional assumption that the skew-product has a regular element that pointwise fixes a center fiber, we extend to cocycles with values in diffeomorphism groups the results obtained in [KNT] for cocycles with values in Lie groups.

THEOREM 3.2. Let $\Gamma$ be a discrete group with property $(T)$ acting through a $C^{1}$-action $\rho$ on $M \times \mathbb{T}$, where $M$ is a compact connected Riemannian manifold. Let $\mu$ be a smooth probability measure whose support is $M \times \mathbb{T}$. Assume that:

(1) $\rho$ preserves $\mu$;

(2) $\rho$ preserves each set $M_{y}:=M \times\{y\}, y \in \mathbb{T}$;

(3) there is an element $a_{0} \in \Gamma$ such that $\rho\left(a_{0}\right)$ is ergodic (with respect to the measure induced by $\mu$ ) on each set $M_{y}, y \in \mathbb{T}$.

Then any action $\tilde{\rho}: \Gamma \rightarrow \operatorname{Diff}(M \times \mathbb{T})$ which is $C^{1}$-close to $\rho$ and satisfies:

(i) $\tilde{\rho}\left(a_{0}\right)=\rho\left(a_{0}\right)$;

(ii) $\tilde{\rho}(a)\left(M_{y}\right) \cap M_{y} \neq \emptyset$ for all $a \in \Gamma$ and $y \in \mathbb{T}$;

preserves each set $M_{y}$.

Remark. One can replace $\mathbb{T}$ by $\mathbb{R}$, as can be seen from the proof. However, in that case, more care is needed in the definition of the $C^{1}$-closeness. If the fiber is non-compact it is enough that the measure $\mu$ be locally finite.

Using these, one obtains the following:

THEOREM 3.3. Fix $K, L \geq 1$. Let $\Gamma$ be a discrete group with property $(T)$ and $\alpha$ a smooth action of $\Gamma$ on a torus $M=\mathbb{T}^{m}$ such that:

(1) $\alpha$ has a periodic point $x_{0} \in M$ (i.e. the $\alpha$-orbit of $x_{0}$ is finite); denote its stabilizer by $\Gamma_{0} \subset \Gamma$; 
(2) $\alpha \Gamma_{\Gamma_{0}}$ contains a (TNS) abelian action $\alpha_{0}$;

(3) $\alpha \uparrow_{\Gamma_{0}}$ preserves an absolutely continuous probability measure $v$ with support $M$;

(4) the (TNS) abelian action $\alpha_{0}$ is continuously $C^{L^{-}, K}$-locally rigid;

(5) $\alpha \Gamma_{\Gamma_{0}}$ is $C^{L^{-}, 0}$-locally rigid.

(6) $H^{1}\left(\Gamma_{0}, V_{\text {rep }}\right)$, the first cohomology group of $\Gamma_{0}$, is trivial for any finite-dimensional representation rep : $\Gamma_{0} \rightarrow G L(V)$.

Then the action $\rho:=\alpha \times \operatorname{Id}_{\mathbb{T}}$ of $\Gamma$ on $M \times \mathbb{T}$ is $C^{L \vee 2, K^{-}}$-locally rigid, where $a \vee b=$ $\max \{a, b\}$.

\section{Remarks.}

(1) By $[\mathbf{L S}]$ and $[\mathbf{L M M}]$, the $\alpha \Gamma_{\Gamma_{0}}$-invariant absolutely continuous measure $v$ introduced in (3) is automatically smooth.

(2) Conditions (4) and (5) of Theorem 3.3 can be replaced by:

$\left(4^{\prime}\right)$ the $(T N S)$ abelian action $\alpha_{0}$ is continuously $C^{L^{-}, 1}$-locally rigid;

(5') $\alpha \Gamma_{\Gamma_{0}}$ is continuously $C^{L^{-}, K}$-locally rigid.

Proof of Theorem 3.3. Let $\tilde{\rho}$ be a $C^{L \vee 2}$-small perturbation of $\rho$.

We first prove that $\tilde{\rho} \Gamma_{\Gamma_{0}}$ is conjugated to $\rho \Gamma_{\Gamma_{0}}$ through a diffeomorphism $h_{0} \in$ Diff ${ }^{K^{-}}(M \times \mathbb{T})$ which is $C^{1}$-close to the identity. We then show that the diffeomorphism $h_{0}$ actually conjugates $\tilde{\rho}$ to $\rho$ on the whole group. This last argument might be well known, we include it here for the sake of completeness.

By (1), $\rho\left(\Gamma_{0}\right)$ has a pointwise fixed center leaf. One concludes from the theorem of Stowe [St1, St2] and assumption (6) that there is a pointwise fixed center leaf for $\widetilde{\rho}\left(\Gamma_{0}\right)$.

Denote by $\mathcal{A} \subset \Gamma_{0}$ the abelian group that induces the (TNS) action $\alpha_{0}$ and by $\rho_{0}, \widetilde{\rho}_{0}$, the restriction of $\rho$, respectively $\widetilde{\rho}$, to $\mathcal{A}$. Applying Theorem 3.1(c) to the action $\rho_{0}$, one obtains a $C^{1}$-small $C^{K^{-}}$conjugacy $h_{0}$ between $\widetilde{\rho}_{0}$ and $\rho_{0}$, which is $C^{L^{-}}$-small on each horizontal leaf of $\rho_{0}$.

Since $\alpha_{0}$ contains Anosov diffeomorphisms and any Anosov diffeomorphism that preserves a smooth measure is ergodic, it follows that we can apply Theorem 3.2 to the action $\rho \Gamma_{\Gamma_{0}}$ and its perturbation $\left.\hat{\rho}\right|_{\Gamma_{0}}$, where $\hat{\rho}:=h_{0} \circ \widetilde{\rho} \circ h_{0}^{-1}$, by taking $\mu$ to be the product of the smooth measure $v$ and the Lebesgue measure on $\mathbb{T}$.

Therefore, $\left.\hat{\rho}\right|_{\Gamma_{0}}$ preserves each set $M_{y}:=M \times\{y\}, y \in \mathbb{T}$. Using assumption (5), it follows that for each $y \in \mathbb{T}$, the restriction $\left(\left.\hat{\rho}\right|_{\Gamma_{0}}\right)_{y}$ of $\hat{\rho} \Gamma_{\Gamma_{0}}$ to the set $M_{y}$ is conjugated to the action $\alpha \Gamma_{\Gamma_{0}}$ through a homeomorphism $h_{y}$ which is $C^{0}$-close to $\operatorname{Id}_{M}$. But the Anosov actions $\alpha\left\lceil_{\mathcal{A}}\right.$ and $\left(\hat{\rho}\left\lceil_{\mathcal{A}}\right)_{y}\right.$ coincide, hence $h_{y}$ is in the centralizer of $\alpha \Gamma_{\mathcal{A}}$, which is discrete (see [PY]). Thus $h_{y}$ has to be the identity, and therefore $\rho \Gamma_{\Gamma_{0}}=h_{0} \circ \widetilde{\rho} \circ h_{0}^{-1} \Gamma_{\Gamma_{0}}$.

Next, we show that if $h_{0} \circ \tilde{\rho} \circ h_{0}^{-1} \Gamma_{\Gamma_{0}}=\left.\rho\right|_{\Gamma_{0}}$ on a subgroup of finite index $\Gamma_{0} \subset \Gamma$, where $\rho=\alpha \times \operatorname{Id}_{\mathbb{T}}$ and $h_{0} \in \operatorname{Diff}^{1}(M \times \mathbb{T})$ is $C^{0}$-close to the identity, then $h_{0} \circ \widetilde{\rho} \circ h_{0}^{-1}=\rho$ on $\Gamma$ as well. The only property we need is that there is an element $\gamma_{0} \in \Gamma_{0}$ such that $\alpha\left(\gamma_{0}\right)$ is Anosov; the fact that the center leaf is one-dimensional is not relevant.

Without loss of generality, we may assume that $\Gamma_{0}$ is a normal subgroup. Denote $h_{0} \circ \tilde{\rho} \circ h_{0}^{-1}$ by $\hat{\rho}$, as before.

Let $g \in \Gamma$. Then $g \gamma_{0} g^{-1} \in \Gamma_{0}$, and thus $\rho\left(g \gamma_{0} g^{-1}\right)=\hat{\rho}\left(g \gamma_{0} g^{-1}\right)=\hat{\rho}(g) \rho\left(\gamma_{0}\right) \hat{\rho}\left(g^{-1}\right)$ preserves each subset $M_{y}, y \in \mathbb{T}$. Therefore, $\rho\left(\gamma_{0}\right)$ preserves each set $\hat{\rho}\left(g^{-1}\right) M_{y}, y \in \mathbb{T}$, 
$g \in \Gamma$. However, each of these sets is a $C^{1}$-manifold which is not far from $M_{y}$ (at least, on a family of generators of $\Gamma$ ). The only such invariant manifolds have to contain the stable and unstable manifolds of $\rho\left(\gamma_{0}\right)$ (see, e.g., the proof of Lemma 4.1) and therefore must coincide with $M_{y^{\prime}}$ for some $y^{\prime} \in \mathbb{T}$. This means that there is a homomorphism $\Phi: \Gamma \rightarrow \operatorname{Homeo}(\mathbb{T})$ with the property that $\rho(g) M_{y}=M_{\Phi_{g}(y)}, \Phi \uparrow_{\Gamma_{0}} \equiv \operatorname{Id}_{\mathbb{T}}$, and $\Phi$ is $C^{0}$-close to the identity. Then Theorem 4.7 implies that $\Phi \equiv \operatorname{Id}_{\mathbb{T}}$ on $\Gamma$.

Denote, as before, by $\hat{\rho}_{y}$ the restriction of $\hat{\rho}$ to $M_{y}, y \in \mathbb{T}$. For any $g \in \Gamma$,

$$
\alpha(g) \alpha\left(\gamma_{0}\right) \alpha(g)^{-1}=\rho_{y}\left(g \gamma_{0} g^{-1}\right)=\hat{\rho}_{y}\left(g \gamma_{0} g^{-1}\right)=\hat{\rho}_{y}(g) \alpha\left(\gamma_{0}\right) \hat{\rho}_{y}(g)^{-1},
$$

therefore $\hat{\rho}_{y}(g)^{-1} \alpha(g) \in \operatorname{Homeo}(\mathbb{T})$ commutes with $\alpha\left(\gamma_{0}\right)$, which is an Anosov diffeomorphism. The same argument as above shows that $\hat{\rho}_{y}(g)^{-1} \alpha(g)=\operatorname{Id}_{\mathbb{T}}$ on a family of generators, hence $\hat{\rho}_{y}(g)=\alpha(g)$ for all $g \in \Gamma$.

Proof of Theorem 1.2. An example of action satisfying the assumptions of Theorem 3.3 is the standard linear action of $\Gamma$ on $\mathbb{T}^{n}, n \geq 3$, where $\Gamma$ is a subgroup of finite index in $S L(n, \mathbb{Z})$. Indeed, by a theorem of Prasad and Raghunathan $[\mathbf{P R}], \Gamma$ intersects a conjugate of a given $\mathbb{R}$-split Cartan subgroup of $\operatorname{SL}(n, \mathbb{R})$ in a uniform lattice. Hence, the intersection consists of commuting matrices which generate a (TNS) action on $\mathbb{T}^{n}$. Take $L=1$. Condition (4) follows from [KL1], condition (5) from [KLZ], and condition (6) is a corollary of Theorem 2.1 in [Mar]. Thus Theorem 1.2 is a corollary of Theorem 3.3.

Katok and Spatzier have shown in a recent paper [KS2] that if $\alpha_{0}$ is an algebraic Anosov action of $\mathbb{Z}^{\kappa}, \kappa \geq 2$, acting on an infranilmanifold with semisimple linear part such that no non-trivial element of the group has roots of unity as eigenvalues in the induced representation on the abelianization, then $\alpha_{0}$ is $C^{1, \infty}$-locally rigid. Continuity of this local rigidity follows from the proof. In the same paper, they prove the $C^{1, \infty}$-local rigidity of linear Anosov actions of irreducible lattices in linear semisimple Lie groups $G$ all of whose factors have real rank at least two. Moreover, for such a lattice action, there is a maximal abelian $\mathbb{R}$-split subgroup whose action on the abelianization satisfies the above conditions.

Hence, one has the following.

COROLlary 3.4. Let $\Gamma$ be an irreducible lattice in a linear semisimple Lie group $G$ all of whose factors have real rank at least two. If $\alpha: \Gamma \rightarrow \operatorname{Diff}^{\infty}\left(\mathbb{T}^{m}\right)$ is a linear action that contains a (TNS) subaction, then $\alpha \times \mathrm{Id}_{\mathbb{T}}$ is $C^{2, K^{-}}$-locally rigid, for any $K \geq 1$.

By the aforementioned theorem of Prasad and Raghunathan and Remark (3) following the definition of the $(T N S)$ property, given a lattice $\Gamma$ in a semisimple Lie group $G$, the existence of a $(T N S)$ subaction of a linear action $\alpha: \Gamma \rightarrow \operatorname{Diff}\left(\mathbb{T}^{m}\right)$ coming from a representation $\pi: G \rightarrow \operatorname{SL}(m, \mathbb{Z})$ can be decided by computing the weights of $\pi$ on an $\mathbb{R}$-split Cartan subgroup of $G$. The irreducible representations of $\operatorname{SL}(n, \mathbb{R})$ are described by Young tableaus. For example, for $n \geq 3$ and $1 \leq k \leq n-1$, the representations corresponding to the tableaus with one column of height $k \neq n / 2$, or two equal columns of height $k \neq n / 4, n / 3, n / 2,2 n / 3,3 n / 4$, or one line of length $k<n / 2$ contain a (TNS) sub-representation. However, for each given semisimple Lie group, only finitely many irreducible representations do so. 
4. The (TNS) property

In this section we prove Theorem 3.1.

Notice first that given a small perturbation $\tilde{\rho}$ of the $\mathcal{A}$-action $\rho$ and a fixed regular element $a \in \mathcal{A}$, the center lamination of $\tilde{\rho}_{a}$ is preserved by $\tilde{\rho}$, hence it is the center lamination of each regular partially hyperbolic diffeomorphism $\tilde{\rho}_{b}, b \in \mathcal{A}$. Moreover, the leaf-wise conjugacy $H_{a}$ of Theorem 2.1(4) between $\tilde{\rho}_{a}$ and $\rho_{a}$ is a leaf-wise conjugacy for each such $\widetilde{\rho}_{b}$.

Indeed, denote by $\mathcal{L}$ the center foliation of $\rho_{a}$ and by $\widetilde{\mathcal{L}}$ that of $\tilde{\rho}_{a}$. Then, by Theorem 2.1(4) for any $\eta, \xi \in M \times N$ :

$$
\eta \in \widetilde{\mathcal{L}}\left(H_{a}(\xi)\right) \Longleftrightarrow \widetilde{\rho}_{a^{n}}(\eta) \text { never strays away from } \rho_{a^{n}}(\mathcal{L}(\xi)) .
$$

Pick a finite family of regular generators of $\mathcal{A}$, and let $b$ be one element of this family. Then, for $\xi \in M \times N, \widetilde{\rho}_{b}\left(\widetilde{\rho}_{a^{n}}\left(H_{a}(\xi)\right)\right)$ is close to $\rho_{b}\left(\rho_{a^{n}}(\mathcal{L}(\xi))\right)$, uniformly with respect to $n \in \mathbb{Z}$. Since $\rho_{b}$ preserves $\mathcal{L}$, this means that $\tilde{\rho}_{a^{n}}\left(\tilde{\rho}_{b}\left(H_{a}(\xi)\right)\right)$ never strays away from $\rho_{a^{n}}\left(\mathcal{L}\left(\rho_{b}(\xi)\right)\right)$. Therefore, $\widetilde{\rho}_{b}\left(H_{a}(\xi)\right) \in \widetilde{\mathcal{L}}\left(H_{a}\left(\rho_{b}(\xi)\right)\right)$, which shows that $\widetilde{\rho}_{b}$ preserves $\widetilde{\mathcal{L}}$ and $H_{a}$ is a leaf-wise conjugacy between $\left(\widetilde{\rho}_{b}, \widetilde{\mathcal{L}}\right)$ and $\left(\rho_{b}, \mathcal{L}\right)$. The claim now follows from the uniqueness parts of (3) and (4) in Theorem 2.1.

Assume the minimal laminations of $\alpha$ are given by

$$
\mathcal{F}_{i}=\bigcap_{a \in S_{i}} W^{s}(a), \quad S_{i} \subset S \subset \mathcal{A}, i \in I .
$$

Hence $S_{i} \cap S_{j} \neq \emptyset$ for any $i, j \in I$, by the (TNS) property. We can also assume that each $\alpha(a), a \in S$, is hyperbolic.

Let $\tilde{\rho}$ be $C^{1}$-close to $\rho=\alpha \times \mathrm{Id}$, so that Theorem 2.1 can be applied for $r=K+1$.

For $i \in I$ and $b \in S_{i}$ consider the lamination

$$
\tilde{\mathcal{F}}_{i}^{(b)}:=\left(\bigcap_{a \in S_{i}} W^{c s}(\widetilde{a})\right) \bigcap W^{s}(\widetilde{b}),
$$

where $\tilde{a}$ stands for $\widetilde{\rho}(a)$. By Theorem 2.1, the above lamination has $C^{K+1}$ leaves, and its holonomy within $\bigcap_{a \in S_{i}} W^{c s}(\widetilde{a})$ is $C^{K}$.

LEMMA 4.1. If $a, b \in S_{i}$ then $\widetilde{\mathcal{F}}_{i}^{(a)}=\widetilde{\mathcal{F}}_{i}^{(b)}$.

Proof. By the unique integrability of these laminations, it is enough to deal with their tangent distributions. Since the action is abelian, the splittings $T(M \times N)=E^{s}(\widetilde{a}) \oplus$ $\widetilde{E}^{c} \oplus E^{u}(\widetilde{a})=E^{s}(\widetilde{b}) \oplus \widetilde{E}^{c} \oplus E^{u}(\widetilde{b})$ are $\mathcal{A}$-invariant. We claim that

$$
E^{c s}(\widetilde{a}) \cap E^{c s}(\widetilde{b})=\widetilde{E}^{c} \oplus\left[E^{s}(\widetilde{a}) \cap E^{s}(\tilde{b})\right] .
$$

This clearly implies that

$$
E^{s}(\widetilde{a}) \cap\left[E^{c s}(\widetilde{a}) \cap E^{c s}(\tilde{b})\right]=E^{s}(\tilde{b}) \cap\left[E^{c s}(\widetilde{a}) \cap E^{c s}(\tilde{b})\right]=E^{s}(\widetilde{a}) \cap E^{s}(\tilde{b}),
$$

from which the desired conclusion follows.

To prove (4.2), let $\xi \in M \times N$ and $v \in E_{\xi}^{c s}(\widetilde{a}) \cap E_{\xi}^{c s}(\tilde{b})$. Then $v=v_{0}+v_{a}=v_{0}^{\prime}+v_{b}$, with $v_{0}, v_{0}^{\prime} \in \widetilde{E}^{c}, v_{a} \in E^{s}(\widetilde{a}), v_{b} \in E^{s}(\tilde{b})$. If $v_{0}=v_{0}^{\prime}$, we are done. Otherwise, let $w:=v_{b}=\left(v_{0}-v_{0}^{\prime}\right)+v_{a} \in E_{\xi}^{s}(\widetilde{b})$. As $n \rightarrow \infty, w_{n}:=\left(\widetilde{a}_{*}^{n} w\right) /\left\|\widetilde{a}_{*}^{n} w\right\|$ approaches unit vectors in $\widetilde{E}^{c}$, while staying in $E^{s}(\widetilde{b})$. However, the angle between the distributions $E^{s}(\tilde{b})$ and $\widetilde{E}^{c}$ is bounded away from zero, a contradiction. 
By Lemma 4.1, one can denote $\widetilde{\mathcal{F}}_{i}^{(a)}, a \in S_{i}$ by $\widetilde{\mathcal{F}}_{i}$. Moreover,

$$
\widetilde{\mathcal{F}}_{i}=\bigcap_{a \in S_{i}} W^{s}(\widetilde{a})
$$

Definition. Let $X$ be a (compact) Riemannian manifold and $\mathcal{F}$ and $\mathcal{G}$ two $C^{1}$-laminations of $X$.

(a) Given $\delta>0$, by the local leaf of size $\delta$ at $x$ of $\mathcal{F}$, denoted $\mathcal{F}(x, \delta)$, we mean the connected component of $x$ in $\mathcal{F}(x) \cap\left\{y \in X: \operatorname{dist}_{X}(x, y)<\delta\right\}$.

(b) We say that the laminations $\mathcal{F}$ and $\mathcal{G}$ are non-tangent if

$$
\inf _{x \in X} L\left(T_{x} \mathcal{F}, T_{x} \mathcal{G}\right)>0
$$

More generally, a family $\left\{\mathcal{F}_{i}\right\}_{i}$ of $C^{1}$-laminations is jointly non-tangent if

$$
\inf _{x \in X} L\left(T_{x} \mathcal{F}_{i}, \operatorname{span}\left\{T_{x} \mathcal{F}_{j}: j \neq i\right\}\right)>0, \quad \text { for each } i .
$$

(c) We say that the non-tangent laminations $\mathcal{F}$ and $\mathcal{G}$ commute locally if there are $\delta, \varepsilon>0$ such that for any $x \in X, y \in \mathcal{F}(x, \delta)$, and $z \in \mathcal{G}(x, \delta)$, there is exactly one point in $\mathcal{F}(z, \varepsilon) \cap \mathcal{G}(y, \varepsilon)$.

Remarks. Assume that $\mathcal{F}$ and $\mathcal{G}$ are two non-tangent locally commuting $C^{1}$-laminations of a compact manifold.

(1) The following strengthening of the commutativity property holds:

$$
\left\{\begin{array}{l}
\text { there are } \delta, \varepsilon, A>0 \text { such that for any } x \in X, y \in \mathcal{F}(x, \delta) \\
\text { and } z \in \mathcal{G}(x, \delta), \text { the point } w=\mathcal{F}(z, \varepsilon) \cap \mathcal{G}(y, \varepsilon) \text { satisfies } \\
\operatorname{dist}_{X}(x, w) \leq A \max \left\{\operatorname{dist}_{X}(x, y), \operatorname{dist}_{X}(x, z)\right\} .
\end{array}\right.
$$

(2) Given two points $x$ and $y$ in a leaf of $\mathcal{G}$ and a path $\gamma$ in the leaf connecting them, one can define the $\mathcal{G}$-holonomy along $\gamma$ between neighborhoods of $x$ in $\mathcal{F}(x)$ and $y$ in $\mathcal{F}(y)$. This holonomy is a local homeomorphism. (This follows from local commutation for short $\gamma$. In general, decompose $\gamma$ into short segments.)

LEMMA 4.2. For each $i, j \in I$, the laminations $\widetilde{\mathcal{F}}_{i}$ and $\widetilde{\mathcal{F}}_{j}$ commute locally.

Remark. This is the only place where it is important that $M$ is a torus. In this case any pair of minimal laminations of $\alpha$ commute locally. Using the fact that $\widetilde{\rho}$ is a small perturbation of a product map, and the structural stability of partially hyperbolic diffeomorphisms, we show that this property can be lifted to the laminations $\widetilde{\mathcal{F}}_{i}$.

Proof of Lemma 4.2. By the remarks at the beginning of this section, there is a homeomorphism $H \in \operatorname{Homeo}(M \times N)$, which is $C^{K+1}$ in the $N$-direction and induces a leaf-wise conjugacy between the center laminations of $\rho$ and $\tilde{\rho}$. Since $W^{c s}(\rho(a))=$ $W^{s}(\alpha(a)) \times N$, hence the center-stable leaves of $\rho$ are spanned by center leaves, one concludes from the characterization of Theorem 2.1 that $H$ also takes the center-stable laminations of $\rho$ into those of $\widetilde{\rho}$.

By $[\mathbf{F}]$ and $[\mathbf{M}]$, the action $\alpha$ is conjugated through a Hölder homeomorphism to a linear action $\alpha_{0}$. Via this conjugation, stable leaves are mapped to stable leaves. Since the 
stable laminations of $\alpha_{0}$ are affine (i.e. given by translations of linear subspaces; recall that $M=\mathbb{T}^{m}$ ), the laminations $\left\{\mathcal{F}_{i}\right\}$ correspond through this conjugacy to affine foliations, and clearly any two affine foliations on a torus commute locally. We conclude that any two of the laminations $\left\{\mathcal{F}_{i}\right\}$ commute locally as well. Let $\delta_{0}, \varepsilon_{0}$, and $A_{0}$ be constants given by (4.3), chosen to be correct for any pair $\mathcal{F}_{i}$ and $\mathcal{F}_{j}(i, j \in I)$.

Given a subset $B \subset M \times N$, let us denote by $W^{c}(B)$, respectively $\widetilde{W^{c}}(B)$, its saturation with respect to the center lamination of $\rho$, respectively $\widetilde{\rho}$ :

$$
W^{c}(B):=\cup\left\{W^{c}(\xi): \xi \in B\right\}, \quad \widetilde{W^{c}}(B):=\cup\left\{\widetilde{W^{c}}(\xi): \xi \in B\right\} .
$$

Note that $\widetilde{W^{c}}(B)=H\left(W^{c}\left(H^{-1}(B)\right)\right)$.

Let $i, j \in I, \xi \in M \times N, \eta \in \widetilde{\mathcal{F}}_{i}(\xi, \delta), \zeta \in \widetilde{\mathcal{F}}_{j}(\xi, \delta)$. We want to show that $\widetilde{\mathcal{F}}_{i}(\zeta, \varepsilon) \cap \widetilde{\mathcal{F}}_{j}(\eta, \varepsilon)$ has exactly one element. (The values of $\delta$ and $\varepsilon$ will be specified later.)

Consider the saturation by $\widetilde{W^{c}}$ of the above sets. In view of the definition (4.1) of the laminations $\widetilde{\mathcal{F}}_{k}$, there are numbers $\delta^{\prime}, \delta^{\prime \prime}, \varepsilon^{\prime}, \varepsilon^{\prime \prime}, \bar{\delta}^{\prime}, \bar{\delta}^{\prime \prime}, \bar{\varepsilon}^{\prime}, \bar{\varepsilon}^{\prime \prime}>0$ (depending on $\xi, \eta, \zeta, \delta$, and $\varepsilon$ ) such that

$$
\begin{gathered}
\mathcal{F}_{i}\left(x, \delta^{\prime}\right) \times N \subset H^{-1}\left(\widetilde{W^{c}}\left(\widetilde{\mathcal{F}}_{i}(\xi, \delta)\right)\right) \subset \mathcal{F}_{i}\left(x, \bar{\delta}^{\prime}\right) \times N, \\
\mathcal{F}_{j}\left(x, \delta^{\prime \prime}\right) \times N \subset H^{-1}\left(\widetilde{W^{c}}\left(\widetilde{\mathcal{F}}_{j}(\xi, \delta)\right)\right) \subset \mathcal{F}_{j}\left(x, \bar{\delta}^{\prime \prime}\right) \times N, \\
\mathcal{F}_{i}\left(z, \varepsilon^{\prime}\right) \times N \subset H^{-1}\left(\widetilde{W^{c}}\left(\widetilde{\mathcal{F}}_{i}(\zeta, \varepsilon)\right)\right) \subset \mathcal{F}_{i}\left(z, \bar{\varepsilon}^{\prime}\right) \times N, \\
\mathcal{F}_{j}\left(y, \varepsilon^{\prime \prime}\right) \times N \subset H^{-1}\left(\widetilde{W}^{c}\left(\widetilde{\mathcal{F}}_{j}(\eta, \varepsilon)\right)\right) \subset \mathcal{F}_{j}\left(y, \bar{\varepsilon}^{\prime \prime}\right) \times N,
\end{gathered}
$$

where $x=\operatorname{pr}_{M}\left(H^{-1}(\xi)\right), y=\operatorname{pr}_{M}\left(H^{-1}(\eta)\right) \in \mathcal{F}_{i}\left(x, \bar{\delta}^{\prime}\right)$, and $z=\operatorname{pr}_{M}\left(H^{-1}(\zeta)\right) \in$ $\mathcal{F}_{j}\left(x, \bar{\delta}^{\prime \prime}\right)$. (Here $\operatorname{pr}_{M}$ stands for the projection $M \times N \rightarrow M$.) Since the laminations $\left\{\widetilde{\mathcal{F}}_{k}\right\}$ converge in $C^{1}$ to $\left\{\mathcal{F}_{k}\right\}$ as $\widetilde{\rho} \rightarrow \rho$ in $C^{1}$ and $H^{ \pm 1}$ are uniformly continuous, for each $\tilde{\rho}$ $C^{1}$-close to $\rho$ one can first choose $\varepsilon>0$ such that

$$
0<\varepsilon_{1}:=\min _{\eta, \zeta \in M \times N}\left\{\varepsilon^{\prime}, \varepsilon^{\prime \prime}\right\} \leq \max _{\eta, \zeta \in M \times N}\left\{\bar{\varepsilon}^{\prime}, \bar{\varepsilon}^{\prime \prime}\right\}<\varepsilon_{0}
$$

and then $\delta>0$ such that

$$
\max _{\xi \in M \times N}\left\{\bar{\delta}^{\prime}, \bar{\delta}^{\prime \prime}\right\} \leq \min \left\{\delta_{0}, \frac{\varepsilon_{1}}{A_{0}+1}\right\} .
$$

See Figure 1 for what follows.

Therefore $\mathcal{F}_{i}\left(z, \varepsilon^{\prime}\right) \cap \mathcal{F}_{j}\left(y, \varepsilon^{\prime \prime}\right)$ contains exactly one element, hence

$$
\begin{aligned}
\widetilde{W}^{c}\left(\widetilde{\mathcal{F}}_{i}(\zeta, \varepsilon)\right) \cap \widetilde{W}^{c}\left(\widetilde{\mathcal{F}}_{j}(\eta, \varepsilon)\right) & =H\left(H^{-1}\left(\widetilde{W}^{c}\left(\widetilde{\mathcal{F}}_{i}(\zeta, \varepsilon)\right)\right) \cap H^{-1}\left(\widetilde{W}^{c}\left(\widetilde{\mathcal{F}}_{j}(\eta, \varepsilon)\right)\right)\right) \\
& =H\left(\left(\widetilde{\mathcal{F}}_{i}\left(z, \varepsilon^{\prime}\right) \times N\right) \cap\left(\widetilde{\mathcal{F}}_{j}\left(y, \varepsilon^{\prime \prime}\right) \times N\right)\right)
\end{aligned}
$$

consists of exactly one leaf of $\widetilde{W^{c}}$, say $\widetilde{\mathcal{L}}_{0}^{c}$. Let $\tau^{\prime}:=\widetilde{\mathcal{F}}_{i}(\zeta, \varepsilon) \cap \widetilde{\mathcal{L}}_{0}^{c}$ and $\tau^{\prime \prime}:=\widetilde{\mathcal{F}}_{j}(\eta, \varepsilon) \cap \widetilde{\mathcal{L}}_{0}^{c}$ (these intersections contain at most one point, for $\varepsilon$ small enough).

Pick $a \in S_{i} \cap S_{j}$. Then $\tau^{\prime}, \tau^{\prime \prime} \in W^{s}(\xi, \widetilde{a}, \delta+\varepsilon) \cap \widetilde{\mathcal{L}}_{0}^{c}$. Since the last intersection consists of a single point for $\delta+\varepsilon$ small enough, we conclude that $\tau^{\prime}=\tau^{\prime \prime}$.

The (TNS)-property is necessary only to obtain Lemma 4.2. One could then prove the following independent result. 


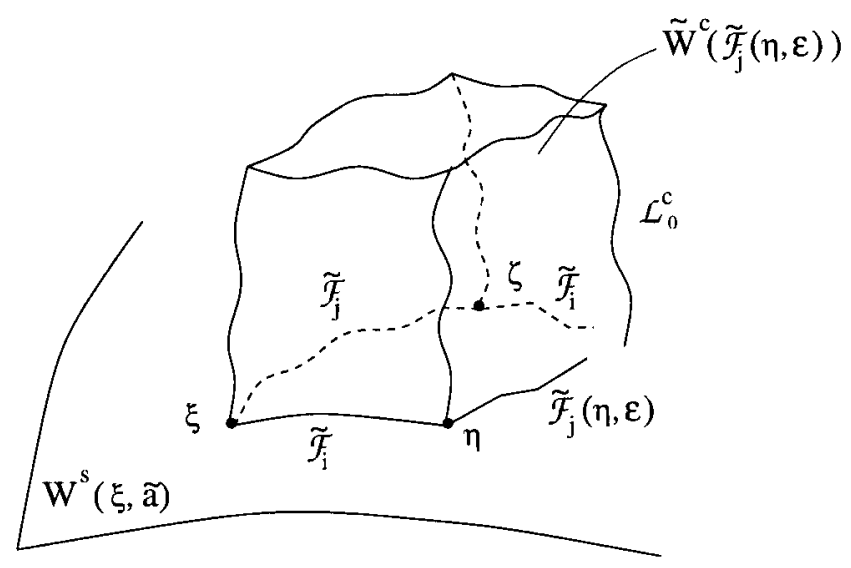

FIGURE 1

THEOREM 4.3. Given a family $\left\{\mathcal{G}_{i}\right\}$ of $C^{1}$-laminations on a compact manifold which are jointly non-tangent and pairwise commute locally, there is a $C^{1}$-lamination $\mathcal{G}$ spanned by them. If the initial laminations are $C^{k}$, then $\mathcal{G}$ is $C^{k^{-}}$.

However, in our case we can take advantage of the fact that $M$ is a torus to simplify the proof.

Lemma 4.4. Let $a \in \mathcal{A}$ be a regular element. If $\tilde{\rho}$ is $C^{1}$-close to $\rho$, then $W^{s}(\widetilde{a})$ and

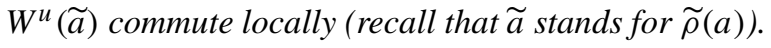

Proof. Assume $W^{s}(\widetilde{a})=\operatorname{span}\left\{\widetilde{\mathcal{F}}_{i}: i \in I^{s}(a)\right\}$ and $W^{u}(\widetilde{a})=\operatorname{span}\left\{\widetilde{\mathcal{F}}_{i}: i \in I^{u}(a)\right\}$, where $I^{S}(a)=\left\{i_{k}: 1 \leq k \leq S\right\}$ and $I^{u}(a)=\left\{j_{k}: 1 \leq k \leq U\right\}$ form a partition of $I$. We claim that for any $\delta_{1}>0$ there is a $\delta_{a}>0$ such that, for $\xi \in M \times N$, each point $\eta \in W^{s}\left(\xi, \widetilde{a}, \delta_{a}\right)$, respectively $\zeta \in W^{u}\left(\xi, \widetilde{a}, \delta_{a}\right)$, can be reached from $\xi$ by juxtaposing segments of lengths at most $\delta_{1}$ of the laminations $\left\{\mathcal{F}_{i}: i \in I^{s}(a)\right\}$, respectively $\left\{\mathcal{F}_{j}: j \in I^{u}(a)\right\}$. That is, there are points $\eta_{i}$ and $\zeta_{j}$ such that

$$
\eta_{i_{1}} \in \widetilde{\mathcal{F}}_{i_{1}}\left(\xi, \delta_{1}\right), \eta_{i_{2}} \in \widetilde{\mathcal{F}}_{i_{2}}\left(\eta_{i_{1}}, \delta_{1}\right), \ldots, \eta=\eta_{i_{S}} \in \widetilde{\mathcal{F}}_{i_{S}}\left(\eta_{i_{S-1}}, \delta_{1}\right)
$$

and

$$
\zeta_{j_{1}} \in \widetilde{\mathcal{F}}_{j_{1}}\left(\xi, \delta_{1}\right), \zeta_{j_{2}} \in \widetilde{\mathcal{F}}_{j_{2}}\left(\zeta_{j_{1}}, \delta_{1}\right), \ldots, \zeta=\zeta_{j_{U}} \in \widetilde{\mathcal{F}}_{j_{U}}\left(\zeta_{j_{U-1}}, \delta_{1}\right)
$$

Indeed, proceed as in Lemma 4.2: consider the saturation of $W^{s}(\xi, \widetilde{a}, \delta)$ by $\widetilde{W}^{c}$, map it via $H^{-1}$ to the corresponding picture for $\rho$ and use the fact that $\alpha$ is conjugated to a linear action to obtain the decomposition of the stable and unstable laminations of $\alpha(a)$ into segments of $\alpha$-minimal laminations. Take the $\rho$-center leaves of the points obtained this way and map them through $H$ to center leaves of $\widetilde{\rho}$. The intersections of these center leaves with $W^{s}(\xi, \tilde{a}, \delta)$ yield the points $\eta_{i}$. By reducing $\delta$ one can obtained the desired conclusion. Apply the same procedure for $W^{u}(\widetilde{a})$.

Pick $\varepsilon>0$ small enough. One can choose $\delta_{1}>0$ (and, therefore, $\delta_{a}$ ) such that Lemma 4.2 and (4.3) imply that the following construction is well defined: given 


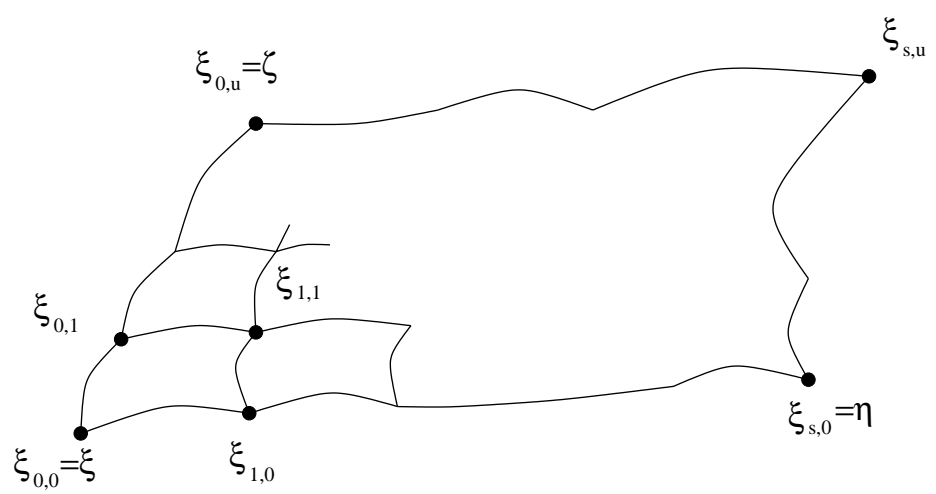

FIGURE 2.

$\xi \in M \times N, \eta \in W^{s}\left(\xi, \widetilde{a}, \delta_{a}\right)$, and $\zeta \in W^{u}\left(\xi, \tilde{a}, \delta_{a}\right)$, let $\eta_{i}, \zeta_{j}$ be as above and define inductively the points $\xi_{k, l}, 0 \leq k \leq S, 0 \leq l \leq U$, by

$$
\begin{gathered}
\xi_{0,0}:=\xi ; \quad \xi_{0, l}:=\zeta_{j l} ; \quad \xi_{k, 0}:=\eta_{i_{k}} ; \\
\xi_{k, l}:=\widetilde{\mathcal{F}}_{i_{k}}\left(\xi_{k-1, l}, \varepsilon\right) \cap \widetilde{\mathcal{F}}_{j_{l}}\left(\xi_{k, l-1}, \varepsilon\right) \quad \text { for } k, l \geq 1 .
\end{gathered}
$$

See Figure 2.

But then $\xi_{S, U} \in W^{u}(\eta, \tilde{a}, U \cdot \varepsilon) \cap W^{s}(\zeta, \tilde{a}, S \cdot \varepsilon)$. Since the stable and unstable laminations of $\tilde{a}$ are non-tangent, for $\varepsilon>0$ small enough this intersection has only one element, thus proving the local commutation of $W^{s}(\widetilde{a})$ and $W^{u}(\widetilde{a})$.

Proof of Theorem 3.1(a). We construct first the lamination $\tilde{\mathcal{H}}$.

Fix a regular element $a \in S$. There is an $\bar{\varepsilon}_{a}>0$ such that the intersection $W^{s}\left(\xi, \tilde{a}, \bar{\varepsilon}_{a}\right) \cap W^{u}\left(\eta, \widetilde{a}, \bar{\varepsilon}_{a}\right)$ has at most one element for any $\xi, \eta \in M \times N$. Choose $\delta_{a}>0, \bar{\varepsilon}_{a}>\varepsilon_{a}>0, A_{a}>1$ satisfying (4.3) applied for $W^{s}(\widetilde{a})$ and $W^{u}(\widetilde{a})$. For $\xi, \eta \in M \times N$, denote

$$
[\xi, \eta]:=W^{s}\left(\xi, \widetilde{a}, \varepsilon_{a}\right) \cap W^{u}\left(\eta, \widetilde{a}, \varepsilon_{a}\right)
$$

(hence, either $[\xi, \eta]$ is empty or it contains one point). Note that local commutativity can be rephrased as

$$
[\xi, \eta] \neq \varnothing \quad \text { and } \quad \operatorname{dist}([\xi, \eta], \xi)<\delta_{a}, \quad \operatorname{dist}([\xi, \eta], \eta)<\delta_{a} \Longrightarrow[\eta, \xi] \neq \emptyset .
$$

Consider $\bar{\delta}_{a}:=\min \left\{\varepsilon_{a}, \delta_{a} /\left(A_{a}+2\right)\right\}$. For $\xi \in M \times N$, define

$$
Q(\xi)=Q\left(\xi, \widetilde{a}, \bar{\delta}_{a}\right):=\left[W^{u}\left(\xi, \widetilde{a}, \bar{\delta}_{a}\right), W^{s}\left(\xi, \widetilde{a}, \bar{\delta}_{a}\right)\right],
$$

where $[U, V]:=\{[u, v]: u \in U, v \in V\}$.

Since the laminations $W^{s}(\widetilde{a})$ and $W^{u}(\widetilde{a})$ are continuous, non-tangent, and commute locally, $Q(\xi)$ with the induced topology from $M \times N$ is homeomorphic to $W^{s}\left(\xi, \tilde{a}_{a}, \bar{\delta}_{a}\right) \times$ $W^{u}\left(\xi, \widetilde{a}, \bar{\delta}_{a}\right)$, i.e. to an open set in $\mathbb{R}^{m}(m=\operatorname{dim} M)$. We claim that the family $\{Q(\xi): \xi \in M \times N\}$ determines plaques of a $C^{0}$-lamination. In view of [R], this is equivalent to showing that

$$
Q(\xi) \cap Q(\eta) \neq \emptyset \Longrightarrow Q(\xi) \cap Q(\eta) \text { is open in both } Q(\xi) \text { and } Q(\eta) .
$$




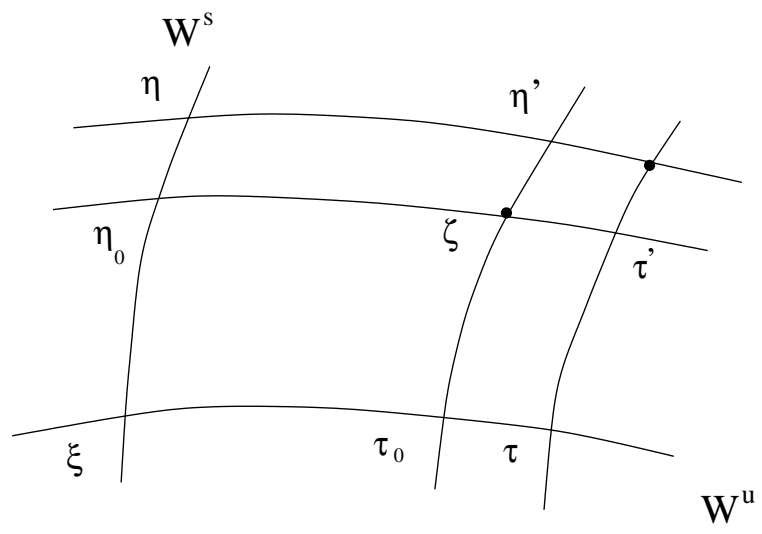

FIGURE 3.

But this follows from (actually, is equivalent to) the local commutativity of the laminations $W^{s}(\widetilde{a})$ and $W^{u}(\widetilde{a})$.

It is enough to show that if $\zeta \in Q(\xi)$, then $\zeta$ belongs to both $\operatorname{Int}_{Q(\xi)}(Q(\xi) \cap Q(\zeta))$ and $\operatorname{Int}_{Q(\zeta)}(Q(\xi) \cap Q(\zeta))$. We will check the first statement, the other being similar. See Figure 3.

Let $\zeta=\left[\tau_{0}, \eta_{0}\right]$, where $\tau_{0} \in W^{u}\left(\xi, \widetilde{a}, \bar{\delta}_{a}\right), \eta_{0} \in W^{s}\left(\xi, \widetilde{a}, \bar{\delta}_{a}\right)$. Note that $\operatorname{dist}_{M \times N}\left(\zeta, \eta_{0}\right), \operatorname{dist}_{M \times N}\left(\tau_{0}, \eta_{0}\right)<\delta_{a}$. Since the holonomy between $W^{s}\left(\xi, \widetilde{a}, \bar{\delta}_{a}\right)$ and $W^{s}\left(\zeta, \widetilde{a}, \bar{\delta}_{a}\right)$ along $W^{u}(\widetilde{a})$ is a local homeomorphism, there is an open neighborhood $V^{s}$ of $\eta_{0}$ in $W^{s}\left(\xi, \tilde{a}, \bar{\delta}_{a}\right)$ such that $\left[\zeta, V^{s}\right] \subset W^{s}\left(\zeta, \widetilde{a}, \bar{\delta}_{a}\right)$. Similarly, there exists an open neighborhood $V^{u}$ of $\tau_{0}$ in $W^{u}\left(\xi, \widetilde{a}, \bar{\delta}_{a}\right)$ with $\left[V^{u}, \zeta\right] \subset W^{u}\left(\zeta, \widetilde{a}, \bar{\delta}_{a}\right)$. Then the set $\left[V^{u}, V^{s}\right]$ is an open neighborhood of $\zeta$ in $Q(\xi)$, and it is also included in $Q(\zeta)$. Indeed, for $\eta \in V^{s}, \tau \in V^{u}$, let $\tau^{\prime}=[\tau, \zeta], \eta^{\prime}=[\zeta, \eta]$. Then $[\tau, \eta]=\left[\tau^{\prime}, \eta^{\prime}\right] \in Q(\zeta)$, as claimed.

We have proved therefore that there is a $C^{0}$-lamination integrating $W^{s}(\widetilde{a})$ and $W^{u}(\widetilde{a})$, hence the family $\left\{\widetilde{\mathcal{F}}_{i}\right\}_{i \in I}$ as well. Call this lamination $\tilde{\mathcal{H}}$. It is clearly $\mathcal{A}$-invariant and horizontal, because so are the laminations used to construct it.

Since it is obtained as the span of two $C^{K+1}$-laminations, its leaves will be $C^{(K+1)^{-}}$, as can be easily seen from the following theorem of Journé (the corollary is a consequence of the proof).

THEOREM 4.5. (Journé $[\mathbf{J}]$ ) Assume given on a manifold two continuous transverse laminations, $\mathcal{F}_{s}$ and $\mathcal{F}_{u}$, with uniformly smooth leaves. If a function $f$ is uniformly $C^{k+\delta_{-}}$ smooth along the leaves of $\mathcal{F}_{s}$ and $\mathcal{F}_{u}$, then $f$ is $C^{k+\delta}$-smooth $(1 \leq k \leq \infty, \delta \in(0,1))$.

COROLlary 4.6. Moreover, if $\mathcal{F}_{s}^{\prime} \rightarrow \mathcal{F}_{s}, \mathcal{F}_{u}^{\prime} \rightarrow \mathcal{F}_{u}, f^{\prime}\left\lceil\mathcal{F}_{u}^{\prime} \rightarrow f \uparrow \mathcal{F}_{u}, f^{\prime}\left\lceil\mathcal{F}_{s}^{\prime} \rightarrow f\left\lceil\mathcal{F}_{s}\right.\right.\right.$ in the $C^{k+\delta}$-topology, then $f^{\prime} \rightarrow f$ in the $C^{k+\delta}$-topology.

Indeed, given a point $\xi \in M \times N$, consider a smooth coordinate system $\chi: U \times V \rightarrow$ $M \times N$ such that in these coordinates a neighborhood of $\xi$ in the leaf $\tilde{\mathcal{H}}(\xi)$ is given by the graph of a function $F: U \rightarrow V$ (here $U$ and $V$ are open subsets of Euclidean spaces). This function is uniformly $C^{K+1}$ along the laminations of $U$ obtained by projecting onto 
$U$ the leaves of $W^{s}(\widetilde{a}) \cap \widetilde{\mathcal{H}}(\xi)$, respectively $W^{u}(\widetilde{a}) \cap \tilde{\mathcal{H}}(\xi)$. Therefore, by Theorem 4.5, $F$ is $C^{(K+1)^{-}}$.

Finally, the holonomy of $\tilde{\mathcal{H}}$ is $C^{K}$ between center leaves because any path along $\widetilde{\mathcal{H}}$ can be realized as a sequence of steps taken alternately inside $W^{c s}(\widetilde{a})$ and $W^{c u}(\widetilde{a})$, and, by Theorem 2.1(2), the stable and unstable holonomies are $C^{K}$ within the leaves of the center-stable, respectively center-unstable, laminations.

Proof of Theorem 3.1(b). Pick some $y_{0} \in N$ and consider the projection $q: M \times N \rightarrow$ $M \equiv M \times\left\{y_{0}\right\}$ along the center foliation of the perturbed action $\widetilde{\rho}$ (which is transversal to both the horizontal lamination $\widetilde{\mathcal{H}}$ and the submanifold $M \times\left\{y_{0}\right\}$ ). Thus $M \times N$ becomes a fiber bundle over $M$, with the fibers $C^{K}$-diffeomorphic to $N$ (by Theorem 2.1(4)). Moreover, this identification between the fibers and $N$ is $C^{k}$-uniformly bounded $(k \leq K)$ with respect to $\widetilde{\rho}$ in a $C^{k}$-small neighborhood of $\rho$.

Due to the way the lamination $\widetilde{\mathcal{H}}$ is constructed, each leaf of it is a covering space of $M$. Given a center leaf $q^{-1}(x)$, consider the holonomy map of $\mathcal{H}, \Delta: \pi_{1}(M, x) \rightarrow$ Homeo $\left(q^{-1}(x)\right)$, obtained as follows: a loop $\gamma \in \Omega(M, x)$ defines the map that associates to $y \in q^{-1}(x)$ the endpoint of the lift beginning at $y$ of $\gamma$ to the leaf $\widetilde{\mathcal{H}}(y)$. Note that, by part (a) of Theorem 3.1, the image of $\Delta$ is actually in $\operatorname{Diff}^{K}\left(q^{-1}(x)\right)$.

The main step is to show that the holonomy map $\Delta$ is trivial.

Assume the fiber $q^{-1}(x)$ is preserved by $\widetilde{\rho}(a)$, for some non-trivial $a \in \mathcal{A}$ (this always happens, because each partially-hyperbolic map has periodic points in the quotient $M \cong(M \times N) / q)$. The $\widetilde{\rho}(a)$-invariance of both $\widetilde{\mathcal{H}}$ and the center laminations imply that

$$
\Delta \circ A_{a}(\gamma)=\phi_{a} \circ \Delta(\gamma) \circ \phi_{a}^{-1}, \quad \gamma \in \pi_{1}(M, x),
$$

where $A_{a} \in \operatorname{Aut}\left(\pi_{1}(M, x)\right)$ is the action induced by $\widetilde{\rho}(a)$ and $\phi_{a}:=\widetilde{\rho}(a) \uparrow_{q^{-1}(x)}$.

In the case when $\Delta$ takes values in a Lie group, equation (4.4) has only the solution $\Delta \equiv I$, provided $\Delta$ and $\phi_{a}$ are close to the identity (see [KNT, Lemma 4.5]). One would expect the same to be true in $\operatorname{Diff}^{K}$, but we were not able to find a proof. However, if we add the condition that $\phi_{a}=\mathrm{Id}$ (i.e. we consider the equation $\Delta \circ A_{a}=\Delta$ ), then this is an easy consequence of the following theorem of Newman $[\mathbf{N}]$, presented here in a version due to Smith [S] (see [B, §9] for a proof).

THEOREM 4.7. (Newman [N]) Let X be a connected topological manifold endowed with a metric. Then there is an $\varepsilon>0$ such that any non-trivial action of a finite group (or compact Lie group) on $X$ has an orbit of diameter larger that $\varepsilon$.

LEMmA 4.8. Let $M=\mathbb{T}^{m}$ and $a \in \operatorname{Diff}(M)$ be an Anosov diffeomorphism that has a fixed point $x_{0} \in M$. Fix a set $T$ of generators of $\pi_{1}\left(M, x_{0}\right) \cong \mathbb{Z}^{m}$. Consider the automorphism $A \in \operatorname{Aut}\left(\pi_{1}\left(M, x_{0}\right)\right) \cong \mathrm{SL}(m, \mathbb{Z})$ induced by $a$.

Given a compact manifold $X$, there is a neighborhood $U$ of the identity in $\operatorname{Homeo}(X)$ with the following property: if $\Delta: \pi_{1}\left(M, x_{0}\right) \rightarrow \operatorname{Homeo}(X)$ is an A-invariant homomorphism (i.e. $\Delta \circ A=\Delta$ ) which maps $T$ into $U$, then $\Delta$ is the trivial homomorphism.

Remark. As in Lemma 4.5 of [KNT], this implies that the same result holds for $a$ an infranilmanifold Anosov diffeomorphism. 


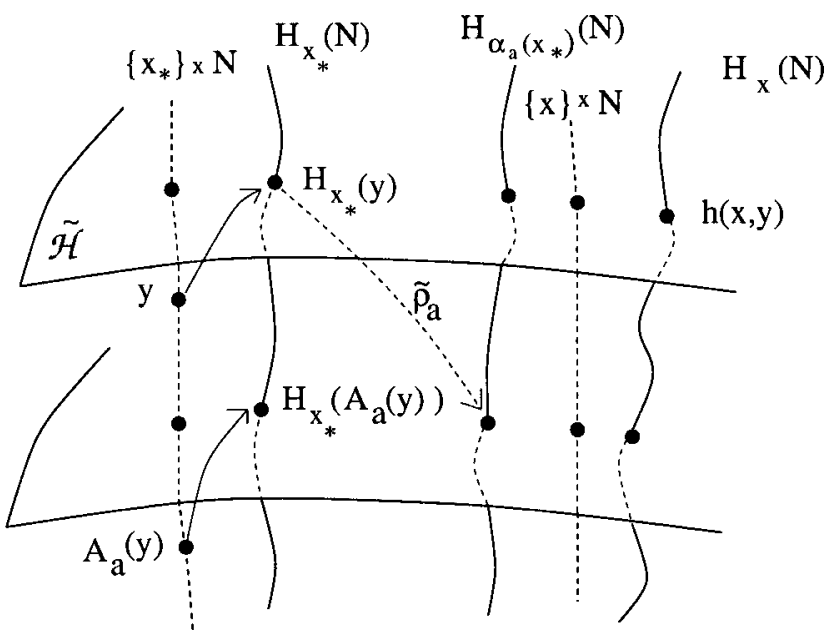

FIGURE 4

Proof of Lemma 4.8. It is enough to consider the case when $T$ is the set $\left\{e_{i}\right\}_{1 \leq i \leq m}$, given by the canonical basis of $\mathbb{Z}^{m}$. Denote $\Delta_{i}:=\Delta\left(e_{i}\right)$.

The equation $\Delta \circ A=\Delta$ implies that $\Delta$ is the identity on the image of $A-I_{m}$. Since $A$ is induced by an Anosov diffeomorphism, 1 is not in the spectrum of $A$, hence $\mathbb{Z}^{m} / \operatorname{Im}\left(A-I_{m}\right)$ is a finite group, of order $c=\operatorname{det}\left(A-I_{m}\right)$. Therefore, $\Delta_{i}^{c}=\operatorname{Id}_{X}$ for each $1 \leq i \leq m$. Let $U$ be a neighborhood of the identity in $\operatorname{Homeo}(X)$ such that Theorem 4.7 can be applied to any cyclic group of order dividing $c$.

Once we know that the holonomy of $\widetilde{\mathcal{H}}$ is trivial, we can construct the desired homeomorphism $h$ as follows. See Figure 4. Let $H \in \operatorname{Homeo}(M \times N)$ be the leafconjugacy between $\rho$ and $\widetilde{\rho}$ given by Theorem 2.1(4). Hence, the center leaves of $\tilde{\rho}$ are given by the images of $H(x, \cdot): N \rightarrow M \times N, x \in M$. Denote $H(x, \cdot)$ by $H_{x}$. Since each $H_{x}$ is a diffeomorphism from $N$ to its image, one can speak of $H_{x}^{-1}$.

Fix a point $x_{*} \in M$ and let

$$
h(x, y):=H_{x}(N) \cap \widetilde{\mathcal{H}}\left(H_{x_{*}}(y)\right) .
$$

That is, $h(x, \cdot) \circ H_{x_{*}}^{-1}$ is the holonomy along $\tilde{\mathcal{H}}$ between the center leaves given by $H_{x_{*}}$ and $H_{x}$. Thus, by part (a) of Theorem 3.1, $h(x, \cdot): N \rightarrow M \times N$ is a $C^{K}{ }_{-}$ diffeomorphism onto its image, as desired. The intersection in the definition is a unique point by Lemma 4.8, and $h$ is a homeomorphism because $\widetilde{\mathcal{H}}$ and the center lamination of $\widetilde{\rho}$ are transverse and give a global 'product structure' on $M \times N$. Note that the leaves of $\widetilde{\mathcal{H}}$ are the images of $h(\cdot, y): M \rightarrow M \times N, y \in N$, and that $h(x, N)=H_{x}(N)$ for each $x \in M$.

It remains to check that $h$ conjugates $\widetilde{\rho}$ to a product action:

$$
\begin{aligned}
\tilde{\rho}_{a}(h(x, y)) & =\widetilde{\rho}_{a}\left(H_{x}(N)\right) \cap \widetilde{\rho}_{a}\left(\widetilde{\mathcal{H}}\left(H_{x_{*}}(y)\right)\right) \\
& =H_{\alpha_{a}(x)}(N) \cap \widetilde{\mathcal{H}}\left(\widetilde{\rho}_{a}\left(H_{x_{*}}(y)\right)\right)=H_{\alpha_{a}(x)}(N) \cap \tilde{\mathcal{H}}\left(H_{x_{*}}\left(A_{a}(y)\right)\right) \\
& =h\left(\alpha_{a}(x), A_{a}(y)\right),
\end{aligned}
$$


where $A_{a} \in \operatorname{Diff}^{K}(N)$ is defined by $h\left(\alpha_{a}\left(x_{*}\right), A_{a}(y)\right)=\widetilde{\rho}_{a}\left(H_{x_{*}}(y)\right)$, hence $\widetilde{\rho}_{a}\left(H_{x_{*}}(y)\right)$ and $H_{x_{*}}\left(A_{a}(y)\right)$ are in the same leaf of $\widetilde{\mathcal{H}}$. (The second equality in (4.6) follows from the fact that $H$ is a leaf-conjugacy and the $\widetilde{\rho}$-invariance of $\widetilde{\mathcal{H}}$.)

The $C^{0}$-convergence of $h$ to $\operatorname{Id}_{M \times N}$ as $\widetilde{\rho}$ converges to $\rho$ in $C^{1}$ follows from Theorem 2.1 and (4.5).

Proof of Theorem 3.1(c). Denote by $\widetilde{M}_{y}$ the image of $M$ through $h(\cdot, y), y \in N$. This is a leaf of $\widetilde{\mathcal{H}}$, hence a $C^{K}$-submanifold of $M \times N$, diffeomorphic to $M$. Denote by $\operatorname{pr}_{M}: M \times N \rightarrow M$ the projection on the first factor. Since $\widetilde{\mathcal{H}}$ is a lamination $C^{K}{ }_{-}$ close to the horizontal foliation of $\rho$, there are uniquely determined $C^{K}$-diffeomorphisms $\phi_{y}: M \rightarrow \widetilde{M}_{y} \subset M \times N$ that are right inverses of $\mathrm{pr}_{M}$; in addition, $y \in N \mapsto \phi_{y} \in C^{K}$ is continuous. In view of Theorem 2.1(3), and Corollary 4.6, $\phi_{y}$ converges in $C^{L^{-}}$to $x \in M \mapsto(x, y) \in M \times N$, uniformly with respect to $y \in N$, as $\widetilde{\rho}$ converges to $\rho$ in $C^{L}$. Under the same conditions, $h_{0}$ converges in $C^{0}$ to $\operatorname{Id}_{M \times N}$, by Theorem 3.1(b). Hence, for each $y \in N, \phi_{y}^{-1} \circ h(\cdot, y)$ conjugates the $\mathcal{A}$-actions $\alpha$ and $\phi_{y}^{-1} \circ \widetilde{\rho}\left\lceil_{\tilde{M}_{y}} \circ \phi_{y}\right.$, and is $C^{0}$-small. Moreover, since the actions are hyperbolic, this is the only conjugacy $C^{0}$-close to $\operatorname{Id}_{M}$. Therefore, since $\alpha$ is continuously $C^{L^{-}, K}$-locally rigid, $\phi_{y}^{-1} \circ h(\cdot, y)$ is $C^{K}$ and varies continuously with $y \in N$ in the $C^{K}$-topology, provided $\tilde{\rho}$ is $C^{L}$-close enough to $\rho$. Thus the same is true for $y \in N \mapsto h(\cdot, y) \in C^{K}(M, M \times N)$.

The fact that $h$ converges to $\operatorname{Id}_{M \times N}$ in $C^{L^{-}}$when $\widetilde{\rho}$ converges to $\rho$ in $C^{L+1}$ follows from the convergence of the holonomy maps (Theorem 2.1(3)), the continuous local rigidity of $\alpha$, and Journé's theorem.

Proof of Theorem 1.1. Theorem 7.1 in [NT1] proves the $C^{K+\mathrm{Lip}, K-3}$-deformation rigidity of $\rho$ for $K \geq 4$. In particular, $\rho$ is $C^{5,1}$-deformation rigid. Let $K \geq 1$ and assume that the smooth action $\widetilde{\rho}$ is $C^{5}$-close enough to $\rho$ so that Theorem 2.1 applies for $\left.\widetilde{\rho}\right|_{\mathcal{A}}$ with $r=K+1$, where $\mathcal{A}$ is an $\mathbb{R}$-split abelian subgroup of $S L(n, \mathbb{Z})$ provided by the theorem of Prasad and Raghunathan. Denote by $h_{0} \in \operatorname{Diff}^{1}\left(\mathbb{T}^{n} \times \mathbb{T}^{d}\right)$ the conjugacy between $\rho$ and $\widetilde{\rho}$ given by [NT1]. Then $\widetilde{\mathcal{H}}$, the image through $h_{0}$ of the horizontal foliation $\mathcal{H}$ of $\rho$, is a $\widetilde{\rho} \uparrow_{\mathcal{A}}$-invariant horizontal foliation which has trivial holonomy. Since it must be spanned by the stable and unstable laminations of $\tilde{\rho}\left\lceil_{\mathcal{A}}\right.$, it is a $C^{(K+1)^{-}}$-lamination. Let $h$ be the conjugacy constructed as at the end of the proof of Theorem 3.1(b). Note that the homomorphism $\pi$ is trivial, because $\rho$ and $\widetilde{\rho}$ are conjugated. Then $h$ is $C^{K}$ along the center direction of $\rho$; along $\mathcal{H}$ one uses the result of [KL1], as was done in [NT1]. Journé's theorem implies that $h$ belongs to $C^{K^{-}}$. Finally, since both $h$ and $h_{0}$ carry $\mathcal{H}$ to $\widetilde{\mathcal{H}}$, they differ by a constant $y$-factor: $h(x, y)=h_{0}(x, \Phi(y))$. Thus $h$ also conjugates $\rho$ and $\widetilde{\rho}$.

\section{5. $\quad$ Property $(T)$}

In this section we prove Theorem 3.2. Although some of the following properties are true in a more general setting, we will state them only for the situation considered by the theorem.

Recall the following consequence of the property $(T)$ (see [HV, Proposition 1.16]):

Assume the group $\Gamma$ has the property $(T)$ relative to the finite set $S \subset \Gamma$. Then, for any $\varepsilon>0$ there is a $\delta_{T}=\delta_{T}(\varepsilon)>0$ such that given a unitary 
representation $\pi: \Gamma \rightarrow \mathcal{U}(H)$ and a $\left(\delta_{T}, S\right)$-invariant vector $\xi \in H, \xi \neq 0$, there exists an invariant vector $\xi^{\prime} \neq 0$ with $\left\|\xi-\xi^{\prime}\right\| \leq \varepsilon\|\xi\|$.

Denote by $\mathbf{1}_{A}: M \times \mathbb{T} \rightarrow \mathbb{R}$ the characteristic function of the set $A \subset M \times \mathbb{T}$.

For $a \in \Gamma$, let $\Delta_{a}:=\partial\left(\tilde{\rho}(a)^{*} \mu\right) / \partial \mu$, the Radon-Nikodym derivative, where $\left(\Phi^{*} \mu\right)(A):=\mu\left(\Phi^{-1}(A)\right)$ for $A \subset M \times \mathbb{T}$ and $\Phi: M \times \mathbb{T} \rightarrow M \times \mathbb{T}$. One obtains a unitary representation $U: \Gamma \rightarrow \mathcal{U}\left(L^{2}(M \times \mathbb{T}, \mu)\right)$ by $U_{a}(f)=\left(f \circ \widetilde{\rho}(a)^{-1}\right) \cdot \Delta_{a}^{1 / 2}$. In the following we use the notation $U_{a}, a \in \Gamma$, for the unitary action described above and $V_{a}$, $a \in \Gamma$, for the unitary action induced by $\rho$ on $L^{2}(M \times \mathbb{T}, \mu)$ (note that since $\rho$ preserves $\left.\mu, V_{a}(f)=f \circ \rho(a)^{-1}\right)$.

Let $\mu_{\mathbb{T}}$ be the measure induced on $\mathbb{T}$ from $\mu$ via the projection $\mathrm{pr}_{\mathbb{T}}: M \times \mathbb{T} \rightarrow \mathbb{T}$ : $\mu_{\mathbb{T}}(A):=\mu(M \times A)$ for $A \subset \mathbb{T}$. Denote by $\mu_{y}$ the conditional measure induced by $\mu$ on $M_{y}, y \in \mathbb{T}: \int_{M \times \mathbb{T}} g d \mu=\int_{\mathbb{T}}\left[\int_{M_{y}} g d \mu_{y}\right] d \mu_{\mathbb{T}}(y)$. Then $\mu_{y}\left(M_{y}\right)=1$ for all $y \in \mathbb{T}$.

Note that if $f \in L^{2}(M \times \mathbb{T}, \mu)$ is invariant under the $\Gamma$-action $U$, then $|f|^{2} d \mu$ is a $\tilde{\rho}$-invariant measure on $M \times \mathbb{T}$. Conditions (2), (3) and (i) of Theorem 3.2 imply that $f$ has to be $\mu_{y}$-a.e. constant on $M_{y}$, for $\mu_{\mathbb{T}}$-a.e. $y \in \mathbb{T}$.

Notation. Denote $\operatorname{dist}_{C^{1}}(\rho, \widetilde{\rho}):=\max \left\{\operatorname{dist}_{C^{1}}(\rho(a), \widetilde{\rho}(a)): a \in S\right\}$. Write $\widetilde{\rho}(a)^{-1}=$ $\left(h_{a}, v_{a}\right)$, where $h_{a}: M \times \mathbb{T} \rightarrow M, v_{a}: M \times \mathbb{T} \rightarrow \mathbb{T}$.

If two functions differ only on a set of $v$-measure zero we will say that they are equal $\bmod v$. We use the same notation for sets that are equal up to a $v$-null set. When there is no possibility of confusion, we will abbreviate the notation for $L^{p}$-spaces.

Remark. Denote by $L^{2}(M \times \mathbb{T})^{\rho}$, respectively $L^{2}(M \times \mathbb{T})^{\tilde{\rho}}$, the vectors of $L^{2}(M \times \mathbb{T})$ that are fixed by the actions $V$, respectively $U$. The hypotheses of Theorem 3.2 imply that

$$
L^{2}(M \times \mathbb{T})^{\widetilde{\rho}} \subset L^{2}(M \times \mathbb{T})^{\rho} \cong L^{2}\left(\mathbb{T}, \mu_{\mathbb{T}}\right) .
$$

The conclusion of the Theorem is that $L^{2}(M \times \mathbb{T})^{\tilde{\rho}}=L^{2}(M \times \mathbb{T})^{\rho}$.

If $L^{2}\left(\mathbb{T}, \mu_{\mathbb{T}}\right)$ were finite dimensional, the result would follow easily from the fact that $\Gamma$ has property $(T)$. In the general case, one needs a generating family of $L^{2}(M \times \mathbb{T})^{\rho}$ which is uniformly almost-invariant for $U$. Note, however, that even if two different measure preserving transformations are close to each other, the unitaries they induce are far apart in the operator norm. Hence, in order to take advantage of the fact that $\tilde{\rho}$ is close to $\rho$, one has to choose these vectors in a special way. Our approach is, roughly speaking, to select them recursively.

Remark. The next two lemmas hold if we replace $\mathbb{T}$ by any compact manifold. Only in Lemma 5.3 is it important that the center direction is one-dimensional.

We begin with some simple estimates.

LEMMA 5.1

(a) If $f \in L^{2}(M \times \mathbb{T}, \mu)$, then

$$
\begin{aligned}
\left\|U_{a}(f)-V_{a}(f)\right\|_{L^{2}} \leq & \left\|f \circ \widetilde{\rho}(a)^{-1}-f \circ \rho(a)^{-1}\right\|_{L^{2}} \cdot\left\|\Delta_{a}^{1 / 2}\right\|_{L^{\infty}} \\
& +\|f\|_{L^{2}} \cdot\left\|\Delta_{a}^{1 / 2}-1\right\|_{L^{\infty}} .
\end{aligned}
$$


(b) If $\phi \in C^{0}(\mathbb{T})$ and $f=\phi \circ \mathrm{pr}_{\mathbb{T}}$ (i.e. $f$ depends only on the second variable, hence is $V$-invariant), then

$$
\left\|f \circ \widetilde{\rho}(a)^{-1}-f\right\|_{L^{2}} \leq\|f\|_{\text {Lip }} \cdot \operatorname{dist}_{C^{0}, \Omega}\left(v_{a}, \operatorname{pr}_{\mathbb{T}}\right) \cdot \mu(\Omega)^{1 / 2},
$$

where $\Omega=\operatorname{supp} f \cup \operatorname{supp}\left(f \circ \widetilde{\rho}(a)^{-1}\right) \subset M \times \mathbb{T}$.

Proof. Both inequalities are straightforward. The first follows from

$$
\begin{aligned}
\left\|U_{a}(f)-V_{a}(f)\right\|_{L^{2}}= & \left\|f \circ \tilde{\rho}(a)^{-1} \cdot \Delta_{a}^{1 / 2}-f \circ \rho(a)^{-1}\right\|_{L^{2}} \\
\leq & \left\|\left(f \circ \widetilde{\rho}(a)^{-1}-f \circ \rho(a)^{-1}\right) \cdot \Delta_{a}^{1 / 2}\right\|_{L^{2}} \\
& +\left\|f \circ \rho(a)^{-1} \cdot\left(\Delta_{a}^{1 / 2}-1\right)\right\|_{L^{2}},
\end{aligned}
$$

using the fact that $\left\|f \circ \rho(a)^{-1}\right\|_{L^{2}}=\|f\|_{L^{2}}$, by the unitarity of $V$.

For the second, notice that both $f \circ \widetilde{\rho}(a)^{-1}$ and $f$ are zero outside $\Omega$, while for $(x, y) \in \Omega \subset M \times \mathbb{T}$

$$
\begin{aligned}
\left|f \circ \widetilde{\rho}(a)^{-1}(x, y)-f(x, y)\right| & =\left|f\left(h_{a}(x, y), v_{a}(x, y)\right)-f\left(h_{a}(x, y), y\right)\right| \\
& \leq\|f\|_{\text {Lip }} \cdot \sup \left\{\operatorname{dist}_{\mathbb{T}}\left(v_{a}(x, y), y\right):(x, y) \in \Omega\right\} .
\end{aligned}
$$

Integrating over $\Omega$ yields the desired conclusion.

The next lemma is probably well known. We include it here for the sake of completeness.

LEMMA 5.2. For any $\varepsilon>0$ there is a $\omega>0$ such that if $\operatorname{dist}_{C^{1}}(\rho, \widetilde{\rho})<\omega$ then: given a $\widetilde{\rho}$-invariant set $\Omega \subset M \times \mathbb{T}$ of positive (finite) measure, there is a $U$-invariant function $\xi_{\Omega} \in L^{2}(M \times \mathbb{T}, \mu)$ which is positive $\mu$-a.e. on $\Omega$ and

$$
\left\|\xi_{\Omega}-1_{\Omega}\right\|_{L^{2}} \leq \varepsilon\left\|1_{\Omega}\right\|_{L^{2}}
$$

Proof. Let $\varepsilon^{\prime}$ be such that $\varepsilon^{\prime} /\left(1-\varepsilon^{\prime}\right)<\varepsilon^{2}$. Since $\Delta_{a} \rightarrow 1$ uniformly for any $a \in \Gamma$ as $\operatorname{dist}_{C^{1}}(\rho, \widetilde{\rho}) \rightarrow 0$, one can find $\omega>0$ such that

$\left\|U_{a}\left(\mathbf{1}_{A}\right)-\mathbf{1}_{A}\right\|_{L^{2}}=\left(\int_{A}\left|\Delta_{a}^{1 / 2}-1\right|^{2} d \mu\right)^{1 / 2} \leq\left\|\Delta_{a}^{1 / 2}-1\right\|_{L^{\infty}} \cdot\left\|\mathbf{1}_{A}\right\|_{L^{2}} \leq \delta_{T}\left(\varepsilon^{\prime}\right)\left\|\mathbf{1}_{A}\right\|_{L^{2}}$

for any $\widetilde{\rho}$-invariant set $A \subset M \times \mathbb{T}$ and all $a \in S$, provided $\operatorname{dist}_{C^{1}}(\rho, \widetilde{\rho})<\omega$. Then, by property $(T)$, the following holds whenever $\operatorname{dist}_{C^{1}}(\rho, \widetilde{\rho})<\omega$ : given a $\widetilde{\rho}$-invariant set $A$ of positive (finite) measure, there is a $U$-invariant function $\xi_{A}^{\prime}$ such that $\operatorname{supp}\left(\xi_{A}^{\prime}\right) \subset A$ and $\left\|\xi_{A}^{\prime}-\mathbf{1}_{A}\right\|_{L^{2}} \leq \varepsilon^{\prime}\left\|\mathbf{1}_{A}\right\|_{L^{2}}$. In particular, $A \backslash \operatorname{supp}\left(\xi_{A}^{\prime}\right)$ is a $\widetilde{\rho}$-invariant set and

$$
\mu\left(A \backslash \operatorname{supp}\left(\xi_{A}^{\prime}\right)\right)^{1 / 2} \leq\left\|\xi_{A}^{\prime}-\mathbf{1}_{A}\right\|_{L^{2}} \leq \varepsilon^{\prime}\left\|\mathbf{1}_{A}\right\|_{L^{2}}=\varepsilon^{\prime} \mu(A)^{1 / 2}
$$

(the condition on the support can be satisfied by restricting to $A$ the $U$-invariant function provided by the property $(T))$.

Let $\Omega_{0}:=\Omega, \xi_{0}:=\xi_{\Omega}^{\prime}$ and define recursively $\Omega_{n}:=\Omega_{n-1} \backslash \operatorname{supp}\left(\xi_{n-1}\right), \xi_{n}:=\xi_{\Omega_{n}}^{\prime}$ as long as $\mu\left(\Omega_{n}\right)>0$. 
Then $\xi_{\Omega}:=\sum_{n \geq 0} \xi_{n}$ has the desired properties. Indeed, $\left\|\mathbf{1}_{\Omega_{n}}\right\|_{L^{2}} \leq \varepsilon^{\prime}\left\|\mathbf{1}_{\Omega_{n-1}}\right\|_{L^{2}}$ by (5.1) and $\left\|\xi_{n}\right\|_{L^{2}} \leq\left(1+\varepsilon^{\prime}\right)\left\|\mathbf{1}_{\Omega_{n}}\right\|_{L^{2}}$, hence $\sum_{n \geq 0} \xi_{n}$ converges in $L^{2}$; the limit is $U$-invariant, and its support is equal to $\Omega$, up to a measure-zero set. Finally,

$$
\begin{aligned}
\left\|\xi_{\Omega}-\mathbf{1}_{\Omega}\right\|_{L^{2}} & =\left(\sum_{n \geq 0}\left\|\xi_{n}-\mathbf{1}_{\Omega_{n} \backslash \Omega_{n+1}}\right\|_{L^{2}}^{2}\right)^{1 / 2} \leq\left(\sum_{n \geq 0}\left\|\xi_{n}-\mathbf{1}_{\Omega_{n}}\right\|_{L^{2}}^{2}\right)^{1 / 2} \\
& \leq\left(\sum_{n \geq 0} \varepsilon^{\prime 2}\left\|\mathbf{1}_{\Omega_{n}}\right\|_{L^{2}}^{2}\right)^{1 / 2} \leq\left(\sum_{n \geq 0}\left(\varepsilon^{\prime}\right)^{n+1}\right)^{1 / 2}\left\|\mathbf{1}_{\Omega}\right\|_{L^{2}} .
\end{aligned}
$$

The main step in the proof is the following.

Lemma 5.3. Assume the hypotheses of Theorem 3.2 hold. Fix $p \geq 1$. There is $\varepsilon_{*}>0$ such that if $\operatorname{dist}_{C^{1}}(\rho, \widetilde{\rho}) \leq \varepsilon_{*}$ then the following holds.

Let $I \subset \mathbb{T}$ be a connected set with a non-empty interior such that $\tilde{\rho}$ preserves $M \times I$. Then there is a measurable function $\psi: M \times I \rightarrow[0, \infty)$ such that $\psi \circ \tilde{\rho}_{a}=\psi \mu$-a.e. for each $a \in \Gamma$ and $\mu\left(\psi^{-1}(\{\lambda\})\right) \leq \mu(M \times I) / p$ for any $\lambda \in \mathbb{R}$.

Proof. We can choose a new Riemannian structure on $\mathbb{T}$ such that $\mathbb{T}$ coincides with $\mathbb{T}$ endowed with the standard metric of volume one, and $\operatorname{vol}_{\mathbb{T}}=\mu_{\mathbb{T}}$. Note that this affects dist $_{C^{0}}$ and dist $C^{1}$ by at most a constant factor. We will assume from now on that this is the original set-up of the lemma.

Replacing $I$ by its closure we may assume without loss of generality that $I$ is closed. Denote $M \times I$ by $\Omega$. Let $\ell=\operatorname{vol}_{\mathbb{T}}(I)=\mu(M \times I)$.

Consider first the case $I \neq \mathbb{T}$. Identify $\Omega$ and $M \times[0, \ell]$, but, for simplicity, keep all the other notation unchanged.

Let $\bar{f}:[0, \ell] \rightarrow[0,1]$ be given by

$$
\bar{f}(y)= \begin{cases}2 y / \ell, & y \in[0, \ell / 2], \\ 2-(2 y / \ell), & y \in[\ell / 2, \ell],\end{cases}
$$

and define $f: M \times[0, \ell] \rightarrow \mathbb{R}$ by $f(x, y)=\bar{f}(y)$.

In the following we compute some $L^{2}$-norms explicitly, but it would be enough to notice to which power of $\ell$ these are proportional; this follows from the behavior of these quantities under rescaling.

Let $a \in S$. Since $M \times[0, \ell]$ is $\widetilde{\rho}$-invariant, for each $x \in M$ the function $v_{a}(x, \cdot)$ maps $[0, \ell]$ into itself, hence it has a fixed point. (Actually, condition (ii) of Theorem 3.2 implies that both endpoints of the interval are fixed.) Therefore,

$$
\begin{aligned}
\sup \left\{\operatorname{dist}_{\mathbb{T}}\left(v_{a}(x, y), y\right):(x, y) \in M \times[0, \ell]\right\} & \leq\left\|v_{a}-\operatorname{pr}_{[0, \ell]}\right\|_{\operatorname{Lip}} \cdot \operatorname{diam}([0, \ell]) \\
& \leq C \operatorname{dist}_{C^{1}}(\rho, \widetilde{\rho}) \cdot \ell,
\end{aligned}
$$

where $C$ does not depend on $\tilde{\rho}$ or $I$. Since supp $f \cup \operatorname{supp}\left(f \circ \widetilde{\rho}(a)^{-1}\right) \subset M \times[0, \ell]$, Lemma 5.1(b) implies that

$$
\left\|f \circ \widetilde{\rho}(a)^{-1}-f\right\|_{L^{2}} \leq C\|f\|_{\text {Lip }} \cdot\left[\operatorname{dist}_{C^{1}}(\rho, \widetilde{\rho}) \cdot \ell\right] \cdot \mu(M \times[0, \ell])^{1 / 2} .
$$


But

$$
\|f\|_{L^{2}}=\left[2 \int_{0}^{\ell / 2}\left(\frac{2}{\ell} t\right)^{2} d t\right]^{1 / 2}=\sqrt{\frac{\ell}{3}}
$$

hence

$$
\left\|f \circ \widetilde{\rho}(a)^{-1}-f\right\|_{L^{2}} \leq C^{\prime}\|f\|_{L^{2}} \operatorname{dist}_{C^{1}}(\rho, \widetilde{\rho}),
$$

where the constant $C^{\prime}$ does not depend on $\tilde{\rho}$ or $I$.

In view of Lemma 5.1(a), the inequality (5.2) shows that if $I \neq \mathbb{T}$ then

$$
\sup _{a \in S} \frac{\left\|U_{a}(f)-f\right\|_{L^{2}}}{\|f\|_{L^{2}}} \rightarrow 0 \quad \text { as } \operatorname{dist}_{C^{1}}(\rho, \widetilde{\rho}) \rightarrow 0, \quad \text { independently of } I .
$$

If $I=\mathbb{T}$ then $\ell=1$ and we consider the function $f: \Omega \rightarrow[0,1]$ as above by identifying $\mathbb{T} \backslash\left\{y_{0}\right\}$ with $(0,1)$ for some arbitrary point $y_{0} \in \mathbb{T}$. Since $\| f \circ \widetilde{\rho}(a)^{-1}-$ $f \|_{L^{\infty}} \leq C^{\prime \prime} \operatorname{dist}_{C^{1}}(\rho, \widetilde{\rho})$ for $a \in S$, the property (5.3) is still valid.

Assume fixed small values $\varepsilon_{1}, \varepsilon_{2}>0$, to be specified later. If $\operatorname{dist}_{C^{1}}(\rho, \widetilde{\rho})$ is small enough (independently of $\ell$ ), then by Lemma 5.2, respectively (5.3) and the property $(T)$, there are $U$-invariant functions $\xi_{\Omega}, \phi \in L^{2}(M \times \mathbb{T}, \mu)$ such that $\xi_{\Omega}>0 \mu$-a.e. on $\Omega$ and

$$
\begin{gathered}
\left\|\xi_{\Omega}-\mathbf{1}_{\Omega}\right\|_{L^{2}} \leq \varepsilon_{2}\left\|\mathbf{1}_{\Omega}\right\|_{L^{2}}, \\
\|f-\phi\|_{L^{2}} \leq \varepsilon_{1}\|f\|_{L^{2}} .
\end{gathered}
$$

We can assume that $\phi \geq 0$, because $|\phi|$ has the same property.

Then $\psi:=\left(\phi \cdot \mathbf{1}_{\Omega}\right) / \xi_{\Omega}$ is $\widetilde{\rho}$-invariant $\bmod \mu$. Since $\psi$ is $\mu$-a.e. preserved by $\tilde{\rho}\left(a_{0}\right)=\rho\left(a_{0}\right)$, it follows from (3) and Fubini's theorem that $\psi$ essentially depends only on the second variable: $\psi(x, y)=\bar{\psi}(y),(x, y) \in M \times[0, \ell] \mu$-a.e. We have to show that $\bar{\psi}$ is not constant on a subset of $I$ of too large measure.

Let $\lambda \in \mathbb{R}$ be such that $\Omega_{*}:=\psi^{-1}(\{\lambda\})$ has positive $\mu$-measure. Since $\Omega_{*}$ is $\rho\left(a_{0}\right)$ invariant $\bmod \mu$, there is a measurable set $I_{*} \subset I$ such that $\Omega_{*}=M \times I_{*}$, again $\bmod \mu$.

From (5.5) and the triangle inequality one obtains that

$$
\begin{aligned}
\varepsilon_{1}\|f\|_{L^{2}} & \geq \| \phi\left\lceil\Omega_{*}-f\left\lceil\Omega _ { * } \| _ { L ^ { 2 } } = \| ( \psi \xi _ { \Omega } ) \left\lceil\Omega_{*}-f\left\lceil\Omega_{*} \|_{L^{2}}\right.\right.\right.\right. \\
& =\| \lambda \xi_{\Omega}\left\lceil\Omega_{*}-f\left\lceil\Omega _ { * } \| _ { L ^ { 2 } } = \| \lambda \left(\xi_{\Omega}\left\lceil\Omega_{\Omega_{*}}-\mathbf{1}_{\Omega_{*}}\right)-\left(\lambda \cdot \mathbf{1}_{\Omega_{*}}-f\left\lceil\Omega_{*}\right) \|_{L^{2}}\right.\right.\right.\right. \\
& \geq \| \lambda \cdot \mathbf{1}_{\Omega_{*}}-f\left\lceil\Omega_{\Omega_{*}}\left\|_{L^{2}}-|\lambda| \cdot\right\| \xi_{\Omega} \Upsilon_{\Omega_{*}}-\mathbf{1}_{\Omega_{*}} \|_{L^{2}},\right.
\end{aligned}
$$

hence, using (5.4),

$$
\varepsilon_{1}\left\|\mathbf{1}_{\Omega}\right\|_{L^{2}} \geq \varepsilon_{1}\|f\|_{L^{2}} \geq \| \lambda \cdot \mathbf{1}_{\Omega_{*}}-f\left\lceil\Omega_{\Omega_{*}}\left\|_{L^{2}}-|\lambda| \cdot \varepsilon_{2}\right\| \mathbf{1}_{\Omega} \|_{L^{2}}\right.
$$

that is

$$
\| \lambda \cdot \mathbf{1}_{\Omega_{*}}-f\left\lceil_{\Omega_{*}}\left\|_{L^{2}} \leq\left(\varepsilon_{1}+|\lambda| \cdot \varepsilon_{2}\right)\right\| \mathbf{1}_{\Omega} \|_{L^{2}} .\right.
$$

But

$$
\| \lambda \cdot \mathbf{1}_{\Omega_{*}}-f\left\lceil_{\Omega_{*}} \|_{L^{2}} \geq \begin{cases}\frac{1}{6}\left(\frac{\left.\mu\left(\Omega_{*}\right)\right)}{\mu(\Omega)}\right)^{3 / 2}\left\|\mathbf{1}_{\Omega}\right\|_{L^{2}}, & |\lambda| \leq 2, \\ \frac{1}{2}|\lambda|\left(\frac{\left.\mu\left(\Omega_{*}\right)\right)}{\mu(\Omega)}\right)^{1 / 2}\left\|\mathbf{1}_{\Omega}\right\|_{L^{2}}, & |\lambda| \geq 2 .\end{cases}\right.
$$


Indeed,

$$
\operatorname{vol}_{\mathbb{T}}(\{y \in I:|\lambda-\bar{f}(y)| \leq \delta\}) \leq 2 \delta \ell,
$$

hence

$$
\begin{aligned}
\| \lambda \cdot \mathbf{1}_{\Omega_{*}}-f\left\lceil_{\Omega_{*}} \|_{L^{2}}\right. & =\left\|\lambda \cdot \mathbf{1}_{I_{*}}-\bar{f} \uparrow_{I_{*}}\right\|_{L^{2}(I)}^{2} \geq \delta^{2} \operatorname{vol}_{\mathbb{T}}\left(I_{*} \backslash\{|\lambda-\bar{f}| \leq \delta\}\right) \\
& \geq \delta^{2}\left(\operatorname{vol}_{\mathbb{T}}\left(I_{*}\right)-2 \delta \ell\right),
\end{aligned}
$$

and the choice $\delta=\operatorname{vol}_{\mathbb{T}}\left(I_{*}\right) /(4 \ell)=\mu\left(\Omega_{*}\right) /(4 \mu(\Omega))$ yields the estimate for the case $|\lambda| \leq 2$. The other case is straightforward.

It is clear that for $\varepsilon_{1}$ and $\varepsilon_{2}$ chosen small enough, the inequalities (5.6) and (5.7) cannot be satisfied simultaneously if $\mu\left(\Omega_{*}\right) / \mu(\Omega) \geq 1 / p$.

Proof of Theorem 3.2. Choose $\widetilde{\rho}$ such that $\operatorname{dist}_{C^{1}}(\rho, \widetilde{\rho}) \leq \varepsilon_{*}$, where $\varepsilon_{*}$ is given by Lemma 5.3 for $p=2$.

Let $B:=\left\{y \in \mathbb{T}: \widetilde{\rho}_{a}\left(M_{y}\right) \neq M_{y}\right.$ for some $\left.a \in \Gamma\right\}$. Since $\widetilde{\rho}$ acts through continuous maps, $B$ is an open set. If $B=\emptyset$, we are done. Assume $B$ is non-void.

The complement of $M \times B$ is $\widetilde{\rho}$-invariant, hence so is $M \times B$. Let $I \subset \mathbb{T}$ be one of the connected components of $B$. Then $M \times I$ is $\widetilde{\rho}$-invariant because, by condition (ii) of Theorem 3.2, $\cup\left\{\widetilde{\rho}_{a}(M \times I): a \in \Gamma\right\} \subset M \times B$ is connected and contains $M \times I$. Let $\psi: M \times I \rightarrow \mathbb{R}$ be the function provided by Lemma 5.3. We will show that there is a connected open non-void set $J_{0} \subset I$ such that $\psi$ is $\mu$-a.e. constant on $M \times J_{0}$. Taking the union of all the connected open sets $J \subset I$ that have this property and contain $J_{0}$, we deduce that there is a maximal open connected set $J_{*} \subset I$ such that $\psi$ is $\mu$-a.e. constant on $M \times J_{*}$.

But then $M \times J_{*}$ is $\widetilde{\rho}$-invariant $\bmod \mu$. Indeed, consider the open $\widetilde{\rho}$-invariant set $\Omega=\cup\left\{\tilde{\rho}_{a}\left(M \times J_{*}\right): a \in \Gamma\right\} \supset M \times J_{*}$. By (ii), $\Omega$ is connected. Then conditions (3) and (i) imply that $\Omega=M \times \mathbb{T}_{0} \bmod \mu$ for some open and connected set $\mathbb{T}_{0} \subset \mathbb{T}$. Since $\psi$ is $\widetilde{\rho}$-invariant, $\psi \uparrow_{\Omega}$ is constant $\mu$-a.e. Thus, by the maximality of $J_{*}$, we conclude that $\mathbb{T}_{0}=J_{*}$, i.e. that $\tilde{\rho}_{a}\left(M \times J_{*}\right)=M \times J_{*} \bmod \mu$ for all $a \in \Gamma$, as claimed.

In particular, the boundary of $M \times J_{*}$ is $\widetilde{\rho}$-invariant. Since $\mu_{\mathbb{T}}\left(J_{*}\right) \leq \mu_{\mathbb{T}}(I) / 2$ by Lemma 5.3, there is a point $y^{\prime} \in I \cap \partial J_{*}$. But then (ii) implies that $M_{y^{\prime}}$ is $\widetilde{\rho}$-invariant, contradicting the connectedness of $I$.

It remains to show that given a measurable function $\psi$ supported on a $\widetilde{\rho}$-invariant set $M \times I$ where $\emptyset \neq I \subset B$ is open, connected and such that $\psi \circ \widetilde{\rho}_{a}=\psi \mu$-a.e. for all $a \in \Gamma$, there is an open connected set $J_{0} \subset I$ such that $\psi$ is $\mu$-a.e. constant on $M \times J_{0}$.

Notice first that in view of the conditions (3) and (i) of Theorem 3.2, by changing $\psi$ on a $\mu$-null set we may assume that $\psi(x, y)=\bar{\psi}(y)$ for any $(x, y) \in M \times I$. Moreover, there is a set $Z \subset M \times I$ of full measure such that for any $a \in \Gamma$ :

$$
\begin{gathered}
\tilde{\rho}_{a}(Z)=Z, \\
\psi\left(\tilde{\rho}_{a}(z)\right)=\psi(z), \quad \text { for all } z \in Z
\end{gathered}
$$

(let $A_{b}:=\left\{z \in M \times I: \psi(z) \neq \psi\left(\widetilde{\rho}_{b}(z)\right\}\right.$ and take $\left.Z:=M \times I \backslash \cup\left\{\widetilde{\rho}_{a}\left(A_{b}\right): a, b \in \Gamma\right\}\right)$. Pick $y \in I$ such that $Z_{y}:=M_{y} \cap Z$ has full $\mu_{y}$-measure in $M_{y}$ and $a \in \Gamma$ such that $\tilde{\rho}_{a}\left(M_{y}\right) \neq M_{y}$. Then $\psi$ is constant on $\widetilde{\rho}_{a}\left(Z_{y}\right)$, hence $\bar{\psi}$ is constant on $J_{0}^{\prime}:=\operatorname{pr}_{\mathbb{T}}\left(\widetilde{\rho}_{a}\left(Z_{y}\right)\right)$, 
which implies that $\psi$ is constant on $M \times J_{0}^{\prime}$. We will show that $J_{0}^{\prime}$ coincides $\bmod \mu_{\mathbb{T}}$ with the connected set $\bar{J}_{0}:=\operatorname{pr}_{\mathbb{T}}\left(\widetilde{\rho}_{a}\left(M_{y}\right)\right)$.

Indeed, let $\gamma:[0,1] \rightarrow M_{y}$ be a $C^{1}$ curve such that $\operatorname{pr}_{\mathbb{T}} \rho_{a}(\gamma([0,1]))=\bar{J}_{0}$. By Sard's theorem, a.e. point of $\bar{J}_{0}$ is a regular value for the mapping $\operatorname{pr}_{\mathbb{T}} \circ \rho_{a} \circ \gamma:[0,1] \rightarrow \bar{J}_{0}$. Assume by contradiction that $\bar{J}_{0} \backslash J_{0}^{\prime}$ has positive measure in $\mathbb{T}$ and let $y_{*} \in \bar{J}_{0} \backslash J_{0}^{\prime}$ be a density point which is also a regular value for $\mathrm{pr}_{\mathbb{T}} \circ \tilde{\rho}_{a} \circ \gamma$. Let $t_{*} \in[0,1]$ be a preimage of $y_{*}$ and $x_{*}:=\gamma\left(t_{*}\right) \in M_{y}$. Then $t_{*}$ is a density point of $A:=\gamma^{-1}\left(M_{y} \backslash Z_{y}\right) \subset[0,1]$.

Since $x_{*}$ is a regular point for $\mathrm{pr}_{\mathbb{T}} \circ \widetilde{\rho}_{a}$, there is a neighborhood $O$ of $x_{*}$ in $M_{y}$ such that $O_{1}:=\left\{x \in O: \operatorname{pr}_{\mathbb{T}} \circ \tilde{\rho}_{a}(x)=y_{*}\right\}$ is a codimension-one submanifold of $M_{y}$. Moreover, by the inverse mapping theorem, for some $\delta>0$ and possibly after shrinking $O$ and $O_{1}$, one can choose around $x_{*}$ local coordinates given by a $C^{1}$-map $\Phi: O_{1} \times\left(t_{*}-\delta, t_{*}+\delta\right) \rightarrow O$ such that if $x \in O$ and $\left|t-t_{*}\right|<\delta$ then

$$
\operatorname{pr}_{\mathbb{T}}\left(\rho_{a}(x)\right)=\operatorname{pr}_{\mathbb{T}}\left(\rho_{a}(\gamma(t))\right) \Longleftrightarrow x \in \Phi\left(O_{1} \times\{t\}\right) .
$$

Therefore, $\Phi\left(O_{1} \times\left\{t \in A:\left|t-t_{*}\right|<\delta\right\}\right) \subset M_{y} \backslash Z_{y}$. Since $\Phi$ is a local diffeomorphism, the left-hand side has a positive $\mu_{y}$-measure, which contradicts our choice of $y$.

Acknowledgements. We would like to thank F. Almgren, G. Forni and A. Katok for helpful discussions.

\section{REFERENCES}

[A] D. V. Anosov. Geodesic flows on closed Riemann manifolds with negative curvature. Proc. Steklov Inst. of Math. 90 (1967).

[B] G. E. Bredon. Introduction to Compact Transformation Groups. Academic Press, 1972.

[F] J. Franks. Anosov diffeomorphisms on tori. Trans. Amer. Math. Soc. 145 (1969), 117-124.

[GS] E. Goetze and R. Spatzier. Smooth classifications of Cartan actions of higher rank semisimple Lie groups and their lattices. Ann. Math. 150 (1999), 743-773.

[HV] P. de la Harpe and A. Valette. La propriété $(T)$ de Kazhdan pour les groupes localement compacts. Astérisque 175 (1989).

[H] S. Hurder. Rigidity of Anosov actions of higher rank lattices. Ann. Math. 135(2) (1992), 361-410.

[HPS] M. Hirsch, C. Pugh and M. Shub. Invariant Manifolds (Lecture Notes in Mathematics, 583). Springer, 1977.

[J] J.-L. Journé. A regularity lemma for functions of several variables. Revista Matematica Iberoamericana 4(2) (1988), 187-193.

[KL1] A. Katok and J. Lewis. Local rigidity for certain groups of toral automorphisms. Israel J. Math. 75 (1992), 203-242.

[KL2] A. Katok and J. Lewis. Global rigidity results for lattice actions on tori and new examples of volume preserving actions. Israel J. Math. 93 (1996), 253-280.

[KLZ] A. Katok, J. Lewis and R. Zimmer. Cocycle superrigidity and rigidity of lattice actions on tori. Topology 35 (1996), 27-38.

[KS1] A. Katok and R. Spatzier. First cohomology of Anosov actions of higher rank abelian groups and applications to rigidity. Inst. Hautes Etudes Sci. Publ. Math. 79 (1994), 131-156.

[KS2] A. Katok and R. Spatzier. Differential rigidity of Anosov actions of higher rank abelian groups and algebraic lattice actions. Proc. Steklov Inst. Math. 216 (1997), 292-319.

[KS3] A. Katok and R. Spatzier. Invariant measures for higher-rank hyperbolic abelian actions. Ergod. Th. \& Dynam. Sys. 16 (1996), 751-778. 
[KNT] A. Katok, V. Niţică and A. Török. Nonabelian cohomology of abelian Anosov actions. Ergod. Th. \& Dynam. Sys. 20 (2000), 259-288.

[LS] A. Livsic and J. Sinai. On invariant measures compatible with the smooth structure for transitive U systems. Sov. Math.-Dokl. 13(6) (1972), 1656-1659.

[LMM] R. de la Llave, J. M. Marco and R. Moriyon. Canonical perturbation theory of Anosov systems and regularity results for Livsic cohomology equation. Ann. Math. 123 (1986), 537-612.

[M] A. Manning. There are no new Anosov diffeomorphisms on tori. Amer. J. Math. 96 (1974), 422 429.

[Mar] G. A. Margulis. Discrete Subgroups of Semisimple Lie Groups. Springer, 1991.

[MQ] G. A. Margulis and N. Qian. Local rigidity of weakly hyperbolic actions of higher real rank semisimple Lie groups and their lattices. Preprint, 1997.

[N] M. H. A. Newman. A theorem on periodic transformations of spaces. Q. J. Math. Oxford Ser. 2 (1931), 1-9.

[NT1] V. Niţică and A. Török. Cohomology of dynamical systems and rigidity of partially hyperbolic actions of higher-rank lattices. Duke Math. J. 79(3) (1995), 751-810.

[PY] J. Palis and J. -C. Yoccoz. Centralizers of Anosov diffeomorphisms on tori. Ann. Sci. Ecole Norm. Sup. 22 (1989), 99-108.

[PR] G. Prasad and M. S. Raghunathan. Cartan subgroups and lattices in semisimple groups. Ann. Math. 96 (1972), 296-317.

[PSW] Ch. Pugh, M. Shub and A. Wilkinson. Hölder foliations. Duke Math. J. 86(3) (1997), 517-546.

[QY1] N. Qian and C.-B. Yue. Local rigidity of Anosov higher rank lattice actions. Ergod. Th. \& Dynam. Sys. 18 (1998), 687-702.

[QY2] N. Qian and C.-B. Yue. Local rigidity of discrete group actions on compact quotients of $S L(n, \mathbb{R})$. Math. Res. Letters to appear.

[R] B. L. Reinhart. Differential Geometry of Foliations. Springer, 1983

[S] P. A. Smith. Transformations of finite period, III. Newman's theorem. Ann. Math. 42 (1941), 446458.

[St1] D. Stowe. The stationary set of a group action. Proc. Amer. Math. Soc. 79 (1980), 139-146.

[St2] D. Stowe. Stable orbits of differentiable group actions. Trans. Amer. Math. Soc. 277 (1983), 665684 .

[Z] R. Zimmer. Actions of semisimple groups and discrete subgroups. Proc. Int. Congress of Mathematicians (1986). Amercan Mathematical Society, Providence, RI, 1987, pp. 1247-1258. 